RICE UNIVERSITY

\title{
A Closed-Loop Model of the Ovine Cardiovascular System
}

by

\section{Junhui Qian}

\author{
A Thesis Submitted \\ in Partial Fulfillment of the \\ REQUIREMENTS FOR THE DEGREE \\ MASTER OF SCIENCE
}

Approved, Thesis CommitTee:

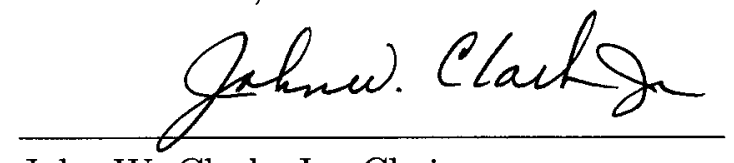

John W. Clark, Jr., Chair

Professor of Electrical and Computer

Engineering

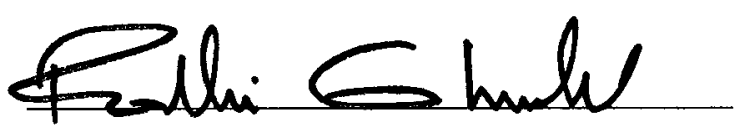

Fathi Ghorbel

Associate Professor of Mechanical

Engineering

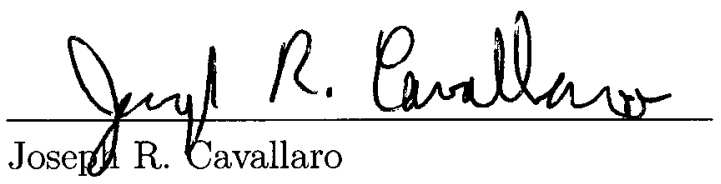

Professor of Electrical and Computer

Engineering and Computer Science

Houston, Texas

February, 2003 
UMI Number: 1414388

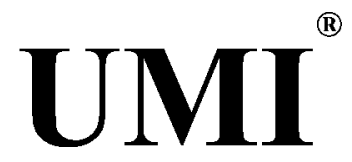

UMI Microform 1414388

Copyright 2003 by ProQuest Information and Learning Company.

All rights reserved. This microform edition is protected against unauthorized copying under Title 17, United States Code.

ProQuest Information and Learning Company 300 North Zeeb Road

P.O. Box 1346

Ann Arbor, MI 48106-1346 


\title{
ABSTRACT
}

\section{A Closed-Loop Model of the Ovine Cardiovascular System}

\author{
by
}

\author{
Junhui Qian
}

The conscious sheep is an important large animal model for the study of human cardiovascular and cardiopulmonary system. In this study we develop a closed-loop mathematical model of its cardiovascular system. A distributed approach is taken in describing the systemic circulation, which is divided into cerebral, coronary, foreleg, thoracic, abdominal, and hind-limb circulations. Nonlinear aspects of the systemic venous system are described, which include nonlinear pressure-volume characteristics of small and large veins and pressure-operated valves in large veins. The complete integrated model mimics typical steady-state hemodynamic data in the supine position. It is also used to predict the blood volume shifts and hemodynamic changes that accompany standing up. These include the short-term neurally mediated cardiovascular response to the orthostatic stress. Additional studies predict the circulatory response to an increased afterload (balloon inflation) presented to the right ventricle. This model is further used to predict the response of the ovine cardiovascular system 
to the implantation of the PAL (Para-corporeal Artificial Lung) device and to test the putative effectiveness of different PAL device designs. 


\section{Acknowledgments}

I am very thankful to Dr. John W. Clark, Jr. for his guidance throughout my MS study. I also thank Kun Lu, from whom I have received a lot of timely help and valuable inspiration. I also thank Dr. Fathi Ghorbel, Dr. Joseph B. Zwischenberger, and Dr. Akhil Bidani. They, together with Dr. Clark, made various research opportunities possible to me, and I have enjoyed.

Heartfelt thanks go to Liang Sun, Yao Wang, Chuan Luo, Limin Chen, and Jin Yang. With them, my life in Rice is not without friendship. Thanks also goes to Wei An. One year of love and despair, however ephemeral and painful, will bring more maturity to both individuals.

Deepest appreciation goes to my parents, Jinfang Qian and Xiaolan Jiang. I would have achieved nothing without their love, support, and expectations. 


\section{Contents}

$\begin{array}{lll}\text { Abstract } & \text { ii }\end{array}$

Acknowledgments

List of Figures viii

List of Tables $\quad$ xiii

1 Introduction $\quad 1$

1.1 Cardiovascular Modeling: A Literature Review . . . . . . . . . . . . 1

1.2 Modeling Objectives . . . . . . . . . . . . . . . 2

1.3 Physiology Background . . . . . . . . . . . . . . . . . . 3

1.3.1 The Heart ................... 3

1.3.2 Vascular System . . . . . . . . . . . . . 4

1.3.3 Control of Arterial Pressure . . . . . . . . . . . . 7

1.3.4 Orthostatic Stress . . . . . . . . . . . . . . . . . 9

1.4 Thesis Outline . . . . . . . . . . . . . . . . . . . 12

2 Whole Body Ovine Cardiovascular Model 13

2.1 Model Development . . . . . . . . . . . . . . . . . . . . . . . 13

2.1 .1 Heart Model . . . . . . . . . . . . . . . . . . . . . . 13

2.1 .2 Circulatory Model . . . . . . . . . . . . . . 13 
2.1.3 Baro-Reflex Control . . . . . . . . . . . . 16

2.2 Simulation Result . . . . . . . . . . . . . . . . . . . 22

2.2.1 Baseline Model Output . . . . . . . . . . . . . . 22

2.2 .2 Adaptation to Orthostatic Stress . . . . . . . . . . . 23

2.2.3 Intra Pulmonary Artery Balloon Inflation . . . . . . . . . 24

2.3 Discussion . . . . . . . . . . . . . . . . . . . 27

2.3.1 Simulation of Orthostatic Stress . . . . . . . . . . 28

2.3.2 Rapid Increase in RV Afterload . . . . . . . . . . . . . . . 29

$3 \quad$ PAL Modeling and Virtual Testing 32

3.1 Development of PAL Device Model . . . . . . . . . . . . . . . 32

3.2 Hemodynamics after Implantation of Baseline-Design PAL . . . . . . 34

3.3 Sensitivity of PAL Design Parameters . . . . . . . . . . . . . . 37

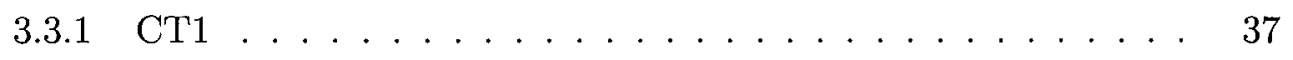

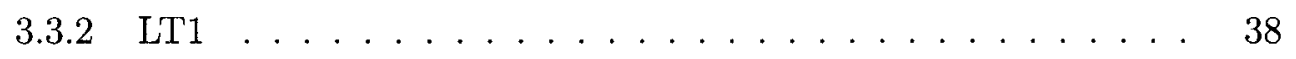

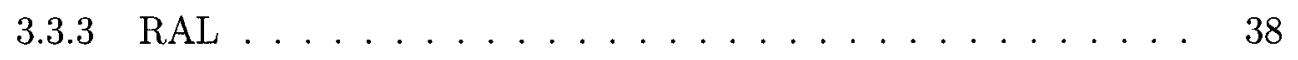

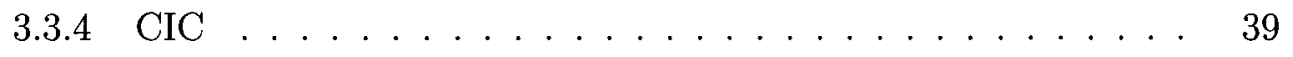

3.4 Discussion . . . . . . . . . . . . . . . . . . 41

3.4.1 The Length of the Inlet Tube . . . . . . . . . . 43

3.4.2 The Resistance of the PAL Device (RAL) . . . . . . . . 43

3.4.3 The Compliance of the Inflow Chamber (CIC) . . . . . . . 44 
$\begin{array}{lll}4 & \text { Summary } & 46\end{array}$

4.1 Cardiovascular Modeling . . . . . . . . . . . . . 46

4.2 Physiological Analysis _. . . . . . . . . . . . . . . . 47

A Heart Muscle Work and Energy Efficiency Index 48

$\begin{array}{ll}\text { B Glossary } & 49\end{array}$

$\begin{array}{ll}\text { References } & \mathbf{5 5}\end{array}$ 


\section{List of Figures}

1.1 Phases of the cardiac cycle. Atrial and ventricular contractions are symbolized by shading of the walls. . . . . . . . . . . . . . 4

1.2 Typical structure of circulatory system of a mammal. The image is from http://gened.emc.maricopa.edu/bio/bio181/BIOBK/BioBookcircSYS.html. 5

1.3 Illustration of artery, arterioles, capillary, venule, and vein, and the change of blood pressure, velocity, and the area from artery to vein. Image is from Purves et al., Life: The Science of Biology, 4th Edition, by Sinauer Associates . . . . . . . . . . . . . . . . . . 6

1.4 Functional organization of the baroreflex control . . . . . . 8

1.5 Pressure-Volume curve for artery and vein. . . . . . . . . . . . 11 
2.1 The equivalent hydraulic circuit of the heart model. RA (Right Atrium), RV (Right Ventricle), LV (Left Ventricle), and LA (Left Atrium) are characterized by time-varying elastance curves. The pressure-operated heart valves are modeled by a diode-resistance pair. The RV ejects blood into pulmonary circulation (via pulmonary artery), which drains into LA via pulmonary vein. The LV ejects blood into systemic circulation (via aortic root), which drains into RA via the Vena Cava. All four chambers are subject to intra-thoracic pressure. . . . . . . . .

2.2 A generic coronary circulation model (modified from [4]). $R_{e p}$ and $C_{e p}$ are the resistance and the compliance of the epicardial artery, respectively. $R_{c o r}$ and $C_{c o r}$ are the distal resistance and compliance of coronary circulation, repectively. A diode is used to ensure the unidirectional perfusion of myocardium, which has time-varying pressure $P_{m c}$ related with intra-ventricle pressure by $P_{m c}=K_{1} * P_{v}(t)+K_{2} *$ $E_{v}(t) * V 0_{v}$, where $K_{1}$ and $K_{2}$ are constants, $P_{v}(t)$ is the ventricular pressure, $E_{v}(t)$ is the elastance of the ventricle, and $V 0_{v}$ is the unstressed volume of the ventricle. . . . . . . . . 
2.3 A typical circulatory branch. The direction of blood flow is from the right to the left, entering via an inertance element. Several compliant chambers are represented, including those of the small artery $\left(C_{s a}\right)$, arterioles $\left(C_{a r}\right)$, capillary $\left(C_{c a}\right)$, small vein $\left(C_{s v}\right)$, large vein $\left(C_{v}\right)$, as well as , the corresponding hydraulic resistances $\left(R_{a}, R_{s a}, R_{a r}, R_{c a}\right.$, and $R_{s v}$ ) between these chambers. $R_{v v}$ is the resistance of venous valve, and $R_{v}$ is the volume-dependent resistance of the large vein. $P_{h}$ represents the hydrostatic pressure of the blood column. And $P_{\text {ref }}$ is the reference pressure, which can be intrathoracic, intra-abdominal, or tissue pressure. 15

2.4 The equivalent fluid-mechanical diagram of ovine cardiovascular system. The pulmonary circulation is modified from [2]. The systemic circulation is divided into cerebral, coronary, thoracic, abdominal, foreleg, and hind-limb circulations. . . . . . . . . . . . . . . 17

2.5 The baro-reflex control system(from $[3]$ ) . . . . . . . . . . . 18

2.6 Neural modulation of instantaneous ventricular elastance. Shown in the figure are four different levels of contractility corresponding to different sympathetic efferent frequencies $F_{C O N}$. (From Lu et al [3]) . 
2.7 An illustration of typical P-V relation curves for the systemic arterioles. The active curve describes the P-V relation of the fully contracted arterioles, while the passive curve describes the nerve-abolished arterioles. A scaling factor mediates the actual instantaneous P-V relation between the two extremes. (From Lu et al [3]) . . . . . . . . . 21

2.8 Baseline model output . . . . . . . . . . . . . . . . . . 22

2.9 Hemodynamic adaptation during standing up. The left four panels show the variation of the MAP (Mean Aortic Pressure), heart rate, cardiac output, and peripheral resistance. The right two panel show the adaptation of the heart pumps using P-V relation loops. . . . . .

2.10 Virtual experiment of the Intra-PA Balloon Inflation. From top to bottom, the PA pressure, the PA outflow, the PA impedances of two steady states, P-V loops of both ventricles, RVMW, RVEEI, LVMW, LVEEI are shown. . . . . . . . . . . . . . . . 27

2.11 Volume redistribution under orthostatic stress challenge . . . . . . . 29

2.12 The interaction between RV and pulmonary circulation . . . . . . . 30

2.13 The interaction between RV and LV . . . . . . . . . . . . . 31

3.1 The PAL attachment . . . . . . . . . . . . . 33

3.2 Hydraulic equivalent model of the PAL connected in series with the proximal and distal pulmonary artery. . . . . . . . . . . . 34 
3.3 Hemodynamics after Implantation of Baseline-Design PAL. . . . . . 36

3.4 The sensitivity of right ventricular muscle work (RVMW), the systolic right ventricular pressure (SRVP), and the pulse flow through the PAL device (PQAL) to the variation of the compliance of the PAL device .

3.5 The influence of inflow chamber on the pulmonary impedance. The bold solid line represents the impedance when inflow chamber is not installed, the solid line when a baseline-design $(\mathrm{CIC}=1.0 \mathrm{ml} / \mathrm{mmHg})$ inflow chamber is attached, the dotted line when a very stiff one $(\mathrm{CIC}=0.1 \mathrm{ml} / \mathrm{mmHg})$ is installed, the dash-dotted line when CIC $=0.5 \mathrm{ml} / \mathrm{mmH}$ g, the dashed line when $\mathrm{CIC}=1.5 \mathrm{ml} / \mathrm{mmHg} . .$.

A.1 Muscle Work Calculation Based on P-V Relation. . . . . . . . . . . . 48 


\section{List of Tables}

2.1 Hemodynamic indices . . . . . . . . . . . . . . . . . 23

3.1 Baseline parameter values of PAL device and the grafts . . . . . . . 35

3.2 Hemodynamic changes induced by PAL implantation . . . . . . . . 37

3.3 The effects of inlet tube compliance $(\mathrm{CT} 1) \ldots \ldots . \ldots 38$

3.4 The effects of inlet tube inertance $(\mathrm{LT} 1) \ldots \ldots \ldots$

3.5 The effects of PAL resistance (RAL) . . . . . . . . . . . 40 


\section{Chapter 1 Introduction}

Due to its anatomic similarities with human, the conscious sheep has become a popular large animal model for cardiovascular and cardiopulmonary diseases([29], [30], [32], [34], [35], [36]). It is also used to test cardiovascular([31], [33]) and pulmonary assist devices $([27])$. This study is focused on the development of a mathematical model of ovine cardiovascular system and the application of this model in PAL (Paracorporeal Artificial Lung) testing.

\subsection{Cardiovascular Modeling: A Literature Review}

Since German physiologist Otto Frank published the famous "Windkessel model" of the arterial system in 1899 , many studies have been devoted to more detailed modeling of the cardiovascular system. A complete review of these models is provided in the work of Linkens [5], Coleman [6], Beneken[7] and Skalak [8].

Whole body circulatory models containing both systemic and pulmonic circulations have been developed previously by Beneken and Rideout [15] and Snyder and Rideout [26]. The latter also incorporated additional features such as reflex neural control, nonlinearity, venous valves and intrathoracic pressure modulation. More recently, Santamore and Amoore et al $[10,11,12]$ developed heart models to study

ventricular performance and ventricular interaction. Hardy et al [13] developed a 
multi-compartment circulatory model to simulate short-term blood flow, gas transport and gas exchange. Beyar et al [14] studied the effects of respiration on circulation and cardiopulmonary resuscitation using a mathematical circulatory model. Olansen

et al [2] developed a multi-compartment model of the canine circulatory system using a heart model capable of characterizing ventricular interaction and the mechanical effect of pericardial constraint. And Lu et al [3] in the same group developed a human cardiovascular and cardiopulmonary model, which describes cardiac mechanics, systemic and pulmonary circulatory dynamics, baroreflex control of arterial pressure, lung mechanics, and alveolar gas exchange.

\subsection{Modeling Objectives}

This study extends effort of our group in developing cardiovascular models([1], $[2],[3]$ ). Previous models have described the hemodynamics of the subject (dog or human) in supine position, whereas the current study considers the subject in both supine and standing positions. In this case the blood volume distribution not only depends on the compliance and the unstressed blood volume, but also the relative hydrostatic position of different organs. Hence a more distributed approach is required in modeling systemic circulations, compared with supine models. There is also an attendant increase in computational complexity. However, this expanded model proves much more useful in a variety of orthostatic applications, particularly in studies of orthostatic challenges of human space flight, as well as in artificial lung testing in 
conscious standing or reclining sheep.

\subsection{Physiology Background}

This section is going to provide physiological background of this study. I am going to review heart physiology, the dynamics of cardiovascular system, the baroreflex control of arterial pressure, and the effect of orthostatic stress on hemodynamics.

\subsubsection{The Heart}

Heart is the pump of cardiovascular system, which could be seen as a fluid transport system. It has four chambers, LV (Left Ventricle), RV (Right Ventricle), LA (Left Atrium), and RA (Right Atrium), working together as a reciprocating pump. Phases of the cardiac cycle are shown in Fig. 1.1. When the ventricular muscle is relaxed (a period called diastole), pressures within the veins and atria exceed the pressure in the ventricle, causing blood to flow into the ventricles through atrioventricular valves. At the end of the diastole, atria begin to contract, forcing additional blood into the ventricles. Since there are no valves between the atria and the veins, some of the blood pumped by the contracting atria is forced back into the veins. The the ventricles begin to contract, and there is a rapid increase in ventricular pressure. As the pressure rises above venous pressure, the atrioventricular valves are closed. At this point all four valves of the heart are closed and the ventricles begin a period of isovolumetric contraction. When ventricular pressure finally exceeds that in the 
outflow tract (either the aorta for the left heart or the pulmonary artery for the right heart), the semilunar valves open and blood is ejected. As the ventricular contractile period (systole) ends, the ventricular musculature relaxes and the force exerted on the blood by the ventricle subsides. As the ventricular muscle continues to relax, the outlet valves closes and a second isovolumetric period occurs, isovolumetric relaxation. When ventricular pressures fall below the corresponding atrial pressure, the inlet valves open and the ventricles fill again, beginning another cycle of pumping.
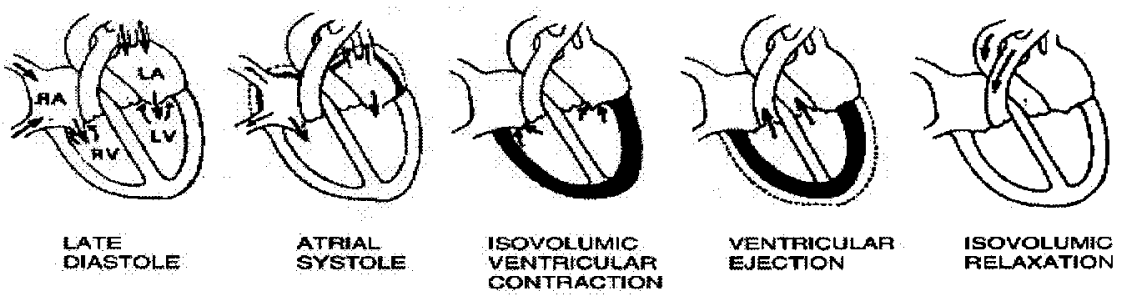

Figure 1.1 Phases of the cardiac cycle. Atrial and ventricular contractions are symbolized by shading of the walls.

\subsubsection{Vascular System}

Structurally, the vascular system can be categorized into pulmonary circulation and systemic circulations, which can further be categorized into cerebral, foreleg, coronary, thoracic, abdominal, hind-leg circulations. Figure 1.2 shows the typical structure of circulatory system of a mammal.

Each circulation contains a serial arrangement of arteries, arterioles, capillaries, 


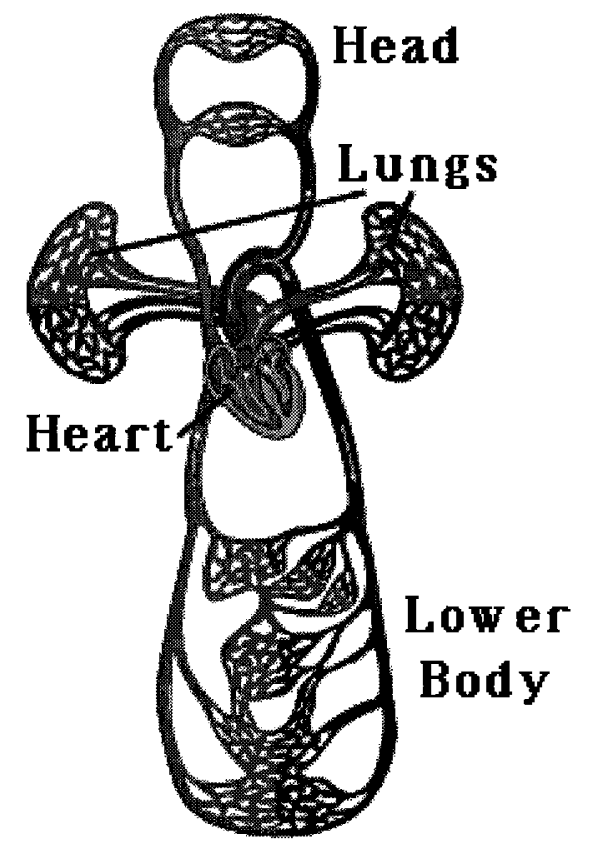

Figure 1.2 Typical structure of circulatory system of a mammal. The image is from http://gened.emc.maricopa.edu/bio/bio181/BIOBK/BioBookcircSYS.html.

venules, and veins (Fig. 1.3). This figure also shows the change in blood pressure, velocity, and cross-section area from artery to vein.

Arteries and arterioles are more than simple tubes that deliver blood from the heart to the tissues. The input from the heart is intermittent and has significant high frequency components. The elastic large arteries and the high resistance arterioles act as s "cushion" or a low frequency filter for this input, converting this intermittent input into a continuous flow driven by the high arterial pressure. Normal arterial pressure pulsates between $120 \mathrm{mmHg}$ (the systolic pressure) and $80 \mathrm{mmHg}$ (the diastolic pressure) for the sheep. Mean pressure is approximately $93 \mathrm{mmHg}$ due 


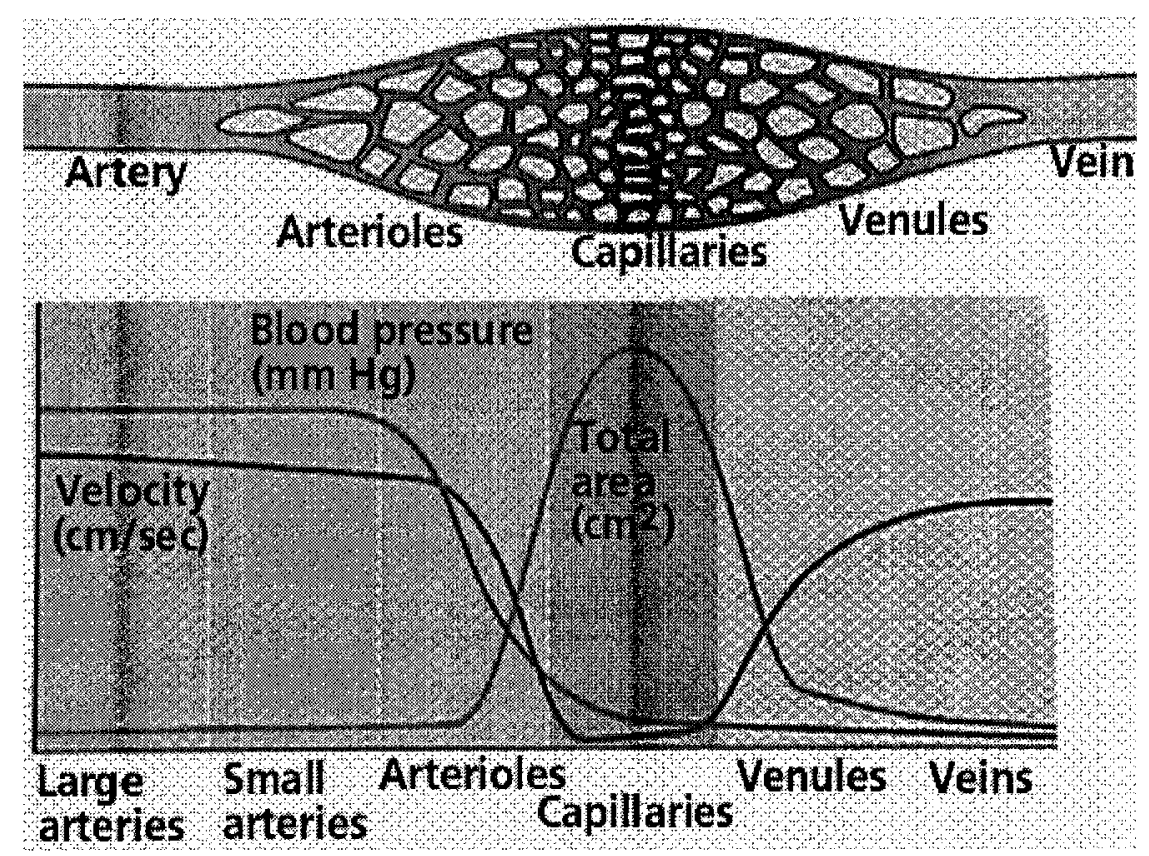

Figure 1.3 Illustration of artery, arterioles, capillary, venule, and vein, and the change of blood pressure, velocity, and the area from artery to vein. Image is from Purves et al., Life: The Science of Biology, 4th Edition, by Sinauer Associates

to the shape of the pulse wave. The difference between the systolic and diastolic pressures is called the pulse pressure.

Capillaries are thin-walled blood vessels in which gas exchange occurs. They are concentrated into highly branched capillary beds, and they offer little resistance to blood flow due to their large cross-section area (Fig. 1.3). Capillary pressure is around $25 \mathrm{mmHg}$.

Venules are smaller veins that gather blood from capillary beds into veins. Venules also offer little resistance to blood flow, and venule pressure is about $13 \mathrm{mmHg}$. Veins 
are thin walled vessels containing blood at low pressures and they carry blood from the organs back to the heart. Veins have valves which ideally allow blood to go to the heart, but not to flow in the opposite direction. As a practical matter, these valves are leaky $([26])$, and they do permit a much reduced backflow. Like most blood vessels, veins are distensible, that is, they have a larger diameter when the pressure inside them is greater and they show the biggest changes in volume per unit change in pressure when the pressures are low. Veins contain some smooth muscle, which is mainly arranged circumferentially and is under sympathetic vasoconstrictor control. For human approximately two thirds of the blood volume resides within the veins, which have a larger diameter than the corresponding arteries.

\subsubsection{Control of Arterial Pressure}

There are four physiological mechanisms to maintain normal blood pressure: baroreflex control, capillary shift mechanism, hormonal responses, kidney and fluid balance mechanisms. The baroreflex control system is illustrated in Fig. 1.4. Baroreflex control is the most rapid regulator of blood pressure. Baroreceptors (pressure sensitive nerve endings) situated in the carotid sinus and the aortic arch sense the arterial pressure, and relay this information to the vasomotor center in the brainstem. A decrease in blood pressure causes activation of the sympathetic nervous system and

results in increased contractility of the heart (beta receptors) and vasoconstriction of both the arterial and venous side of the circulation (alpha receptors), thus partially 
restoring arterial pressure.

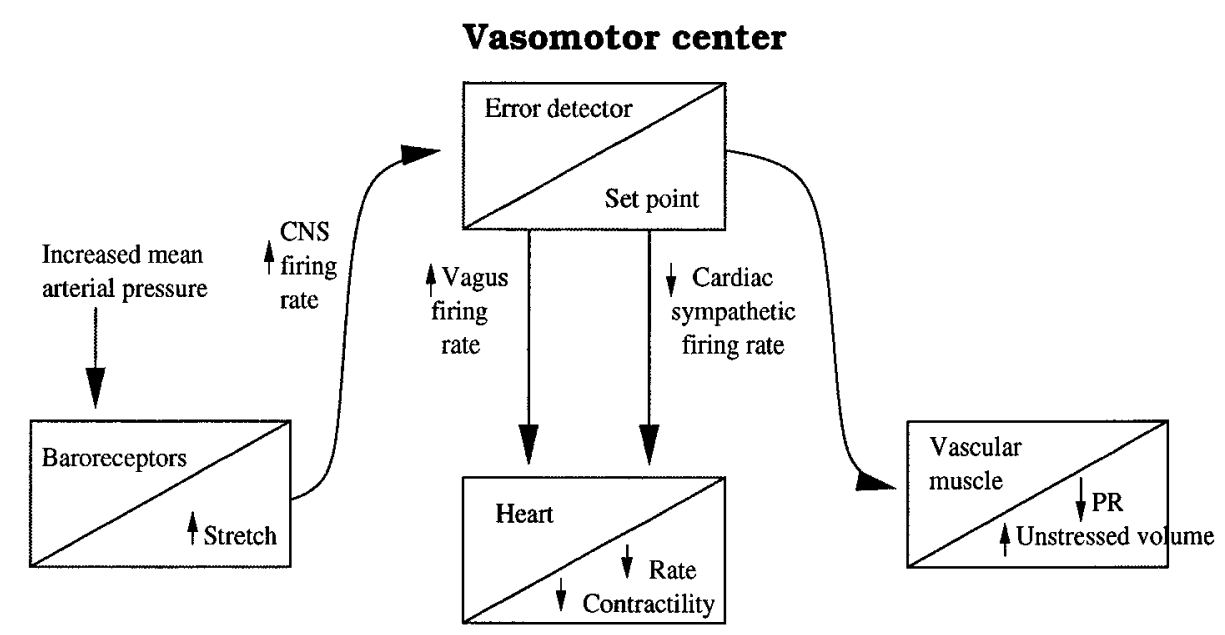

Figure 1.4 Functional organization of the baroreflex control

The "capillary fluid shift" mechanism refers to the filtration of fluid and electrolytes across the porous capillary membrane between the blood and the interstitial body fluid. This filtration is controlled by the capillary blood pressure, the interstitial fluid pressure and the colloid osmotic pressure of the plasma. Low blood pressure results in fluid movement from the interstitial space into the circulation, helping to restore and maintain blood volume and blood pressure.

Hormonal mechanisms exist both for lowering and raising blood pressure. They act in various ways including vasoconstriction, vasodilation and alteration of blood volume. The principal hormones responsible for raising blood pressure are: (a) Epinephrine and norepinephrine secreted from the adrenal medulla in response to 
sympathetic nervous system stimulation. They increase cardiac output and cause vasoconstriction and act very rapidly. (b) Renin and angiotensin production is increased in the kidney when stimulated by hypotension. Angiotensin is converted in the lung to Angiotensin II, which is a potent vasoconstrictor. In addition these hormones stimulate the production of aldosterone from the adrenal cortex which decreases urinary fluid and electrolyte loss from the body. This system is responsible for the long term maintenance of blood pressure but is also activated very rapidly in the presence of hypotension.

The kidneys help to regulate the blood pressure by increasing or decreasing the blood volume and also by the renin-angiotensin system described above. They are the most important organs for the long-term control of blood pressure.

This thesis considers only the short-term control of blood pressure, and hence considers only the baroreflex control of blood pressure.

\subsubsection{Orthostatic Stress}

Increased gravitational force causes marked redistribution of blood volume in accordance with the changes in transmural pressure. When humans stand up, the heart is about 1.2 to $1.5 \mathrm{~m}$ above the feet, and about 75 percent of the blood volume is in compliant veins. Approximately 70 to 75 percent of total blood volume is below the level of the pump and must somehow be driven back to right atrium. This poses stress on the cardiovascular system to function normally. 
Four structural and functional features of the veins help drive venous blood back to right heart, namely, shape of venous Pressure-Volume Curve, venous valve, muscle pump, and respiratory pump.

\section{The Shape of Venous Pressure-Volume Relation Curve}

Figure 1.5 shows an approximate Pressure-Volume relation curve for the vein, as well as one for the artery for comparison. The venous Pressure-Volume relation curve is clearly not linear. It has a low-pressure compliant portion and a high-pressure stiff portion. In low pressure, volume change $(\Delta V)$ results in small change in pressure $\left(\Delta P_{1}\right)$, whereas in high pressure, the same volume change $(\Delta V)$ results large pressure change $\left(\Delta P_{1}\right)$. When a human or a mammal assumes upright position, a large amount of blood volume from thoracic and abdominal circulations will be relocated to the limbs. As a result, venous pressure builds up in limbs, and as this process goes on, the venous Pressure-Volume relation becomes the high-pressure stiff portion, which prevents further blood pooling.

\section{Venous Valves and Muscle Pump}

Venous valves have three important roles in counteracting orthostatic stress. First, with them functioning, the hydrostatic column of blood extending from the veins in the foot back up to the heart is broken up in columns of smaller length, thus reducing venous pressure in the legs. Second, they reduces the rate at which the veins fill 


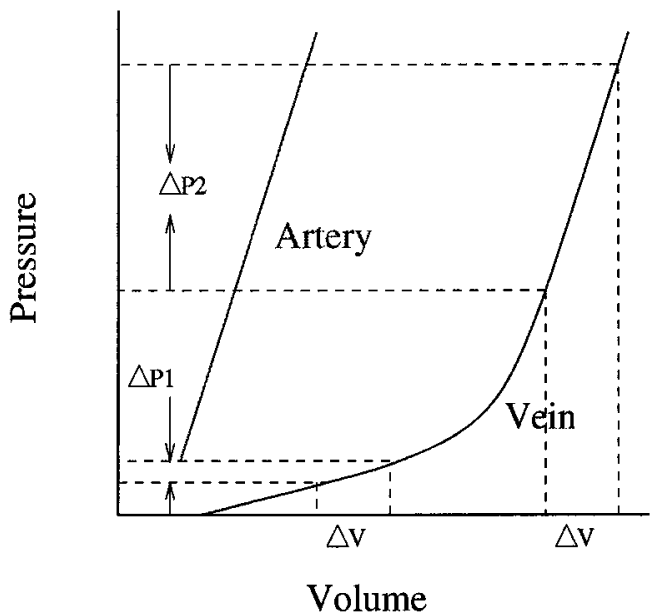

Figure 1.5 Pressure-Volume curve for artery and vein.

up with blood after upright position is assumed. Third, they work with contracting muscles (together they are called "muscle pump", or "venous pump"), to force pooling venous blood flow toward the heart.

\section{Respiration Pump}

The respiration pump refers to the interaction between diaphragm and liver blood volume during inspiration and expiration that help maintain a sustained and smooth venous return. Inspiration in upright posture lowers intrathoracic pressure and increases the pressure gradient between the right atrium and the point where the inferior vena cava enters the thoracic cavity, thus facilitating venous flow into vena cava. During inspiration, descent of diaphragm mechanically stops hepatic venous outflow while inflow continues and liver swells. Expiration raises intrathoracic pressure, thus 
impeding venous flow into the thorax. However, the ascent of diaphragm relieves the compression of the hepatic veins, and venous return is partially maintained by passive release (elastic recoil) of hepatic blood back to the right atrium.

\subsection{Thesis Outline}

Chapter 2 presents the development of the whole-body ovine cardiovascular model and the simulation results under baseline condition, orthostatic stress, and intra pulmonary artery balloon inflation. A discussion based on these results about the model follows.

Chapter 3 tested the implantation of the PAL ( Para-corporeal Artificial Lung) device by developing a lumped parameter model for the device and connecting it serially to the whole-body model. Different designs of the PAL are then examined, with concentration on the effects of individual parameters on right heart energetics and hemodynamics of the PAL device.

A summary of this thesis is offered in Chapter 4. And Appendix A provides the definition and computation method of right ventricular muscle work and energy efficiency. Appendix B provides a glossary for convenience. 


\section{Chapter 2 \\ Whole Body Ovine Cardiovascular Model}

\subsection{Model Development}

This integrated model consists of a heart model that is based on the time-varying elastance concept, a multiple branch circulatory model, and a neural control model that senses changes in arterial blood pressure and responds by changing heart rate, heart muscle contractility, and vasomotor tone.

\subsubsection{Heart Model}

We adopt the heart model (Fig. 2.1) used in previous studies by our group[1], which describes hemodynamic behavior of the left and right ventricles including the effects of preload and afterload, direct ventricular interaction, and the influence of pericardium on pump performance. We also include a generic model of the coronary circulation [4] as part of the heart model, and use two such models to characterize the right and left coronary arteries. This model also considers the influence of transmural pressure on coronary blood flow (Fig. 2.2).

\subsubsection{Circulatory Model}

The systemic circulation is divided into cerebral, coronary, foreleg, thoracic, abdominal, and hind-limb circulations. Each branch has a typical structure analogous to that shown in Fig. 2.3. Although this more distributed approach increases model 


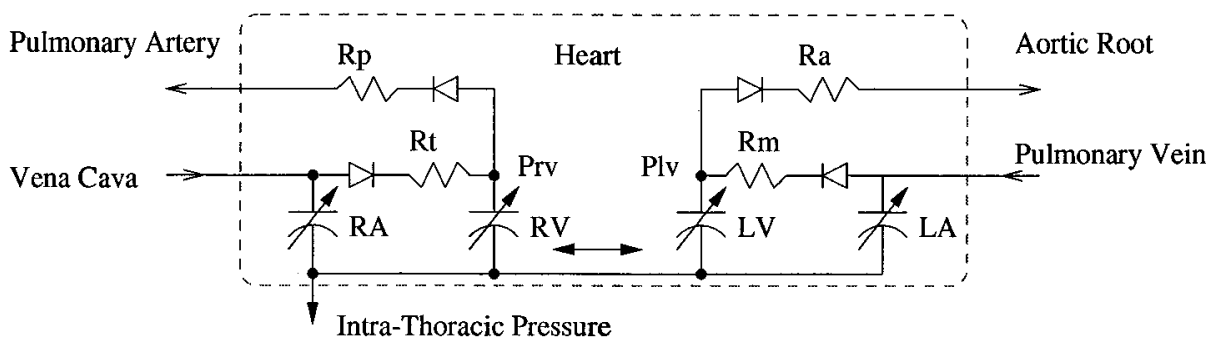

Figure 2.1 The equivalent hydraulic circuit of the heart model. RA (Right Atrium), RV (Right Ventricle), LV (Left Ventricle), and LA (Left Atrium) are characterized by timevarying elastance curves. The pressure-operated heart valves are modeled by a dioderesistance pair. The RV ejects blood into pulmonary circulation (via pulmonary artery), which drains into LA via pulmonary vein. The LV ejects blood into systemic circulation (via aortic root), which drains into RA via the Vena Cava. All four chambers are subject to intra-thoracic pressure.

complexity, it also has the advantage of being able to characterize local pressures, flows, and volumes in major circulatory branches.

We assume a linear pressure-volume $(\mathrm{P}-\mathrm{V})$ relationship for the arterial compliance chambers. However, for small veins the P-V relationships are nonlinear $([25]$, $[26]$ ), and the instantaneous P-V relationship depends on neural sympathetic tone. The large veins are also characterized by nonlinear P-V relations, but their walls are non-muscular and hence are not subject to neural modulation. Importantly, venous circulations contain leaky pressure-operated valves, which are characterized by parallel forward-backward flow resistance models $([26])$. These venous elements are shown as part of Fig. 2.4, which is the equivalent hydraulic circuit diagram of the whole model, and it is these valved return pathways to right heart that enable a sustained venous return, under conditions of orthostatic stress. 


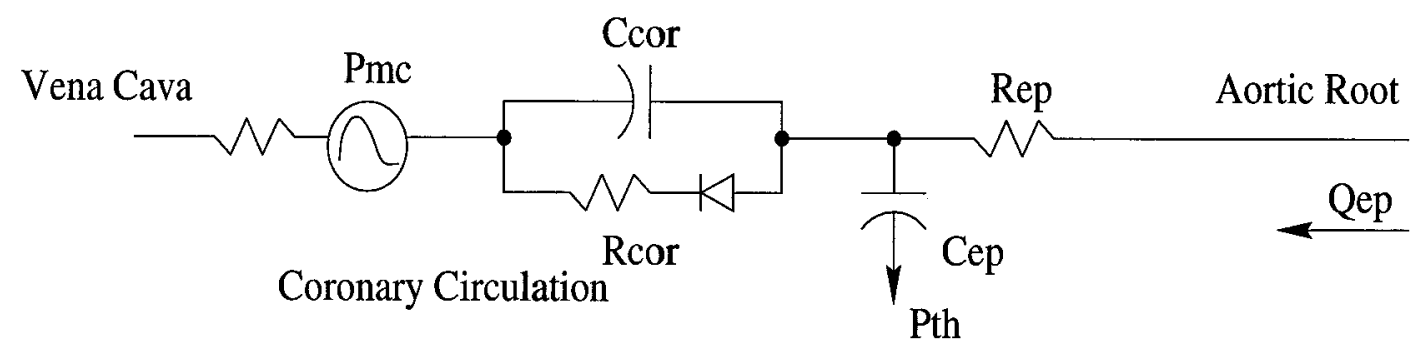

Figure 2.2 A generic coronary circulation model (modified from [4]). $R_{e p}$ and $C_{e p}$ are the resistance and the compliance of the epicardial artery, respectively. $R_{c o r}$ and $C_{c o r}$ are the distal resistance and compliance of coronary circulation, repectively. A diode is used to ensure the unidirectional perfusion of myocardium, which has time-varying pressure $P_{m c}$ related with intra-ventricle pressure by $P_{m c}=K_{1} * P_{v}(t)+K_{2} * E_{v}(t) * V 0_{v}$, where $K_{1}$ and $K_{2}$ are constants, $P_{v}(t)$ is the ventricular pressure, $E_{v}(t)$ is the elastance of the ventricle, and $V 0_{v}$ is the unstressed volume of the ventricle.

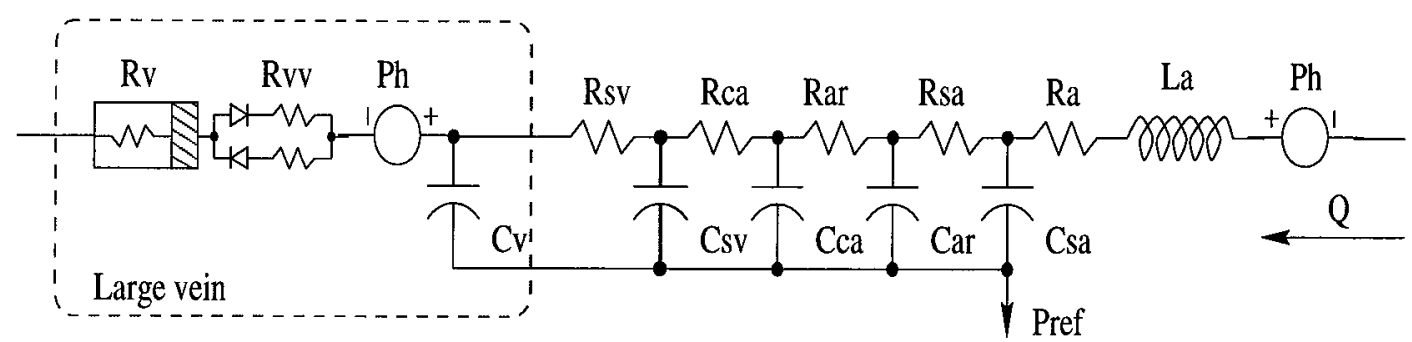

Figure 2.3 A typical circulatory branch. The direction of blood flow is from the right to the left, entering via an inertance element. Several compliant chambers are represented, including those of the small artery $\left(C_{s a}\right)$, arterioles $\left(C_{a r}\right)$, capillary $\left(C_{c a}\right)$, small vein $\left(C_{s v}\right)$, large vein $\left(C_{v}\right)$, as well as, the corresponding hydraulic resistances $\left(R_{a}, R_{s a}, R_{a r}, R_{c a}\right.$, and $R_{s v}$ ) between these chambers. $R_{v v}$ is the resistance of venous valve, and $R_{v}$ is the volumedependent resistance of the large vein. $P_{h}$ represents the hydrostatic pressure of the blood column. And $P_{r e f}$ is the reference pressure, which can be intrathoracic, intra-abdominal, or tissue pressure. 


\subsubsection{Baro-Reflex Control}

We adopt the general framework used in our previous model of the human cardiovascular system [3], to represent the ovine baroreflex control system. Central aortic pressure produced by the model is used as the representative arterial pressure fed to the cardiovascular medullary center (CVMC). Subsequent motor control action changes heart rate, myocardial contractility, and vasomotor tone. These components are shown in Fig. 2.5 . 


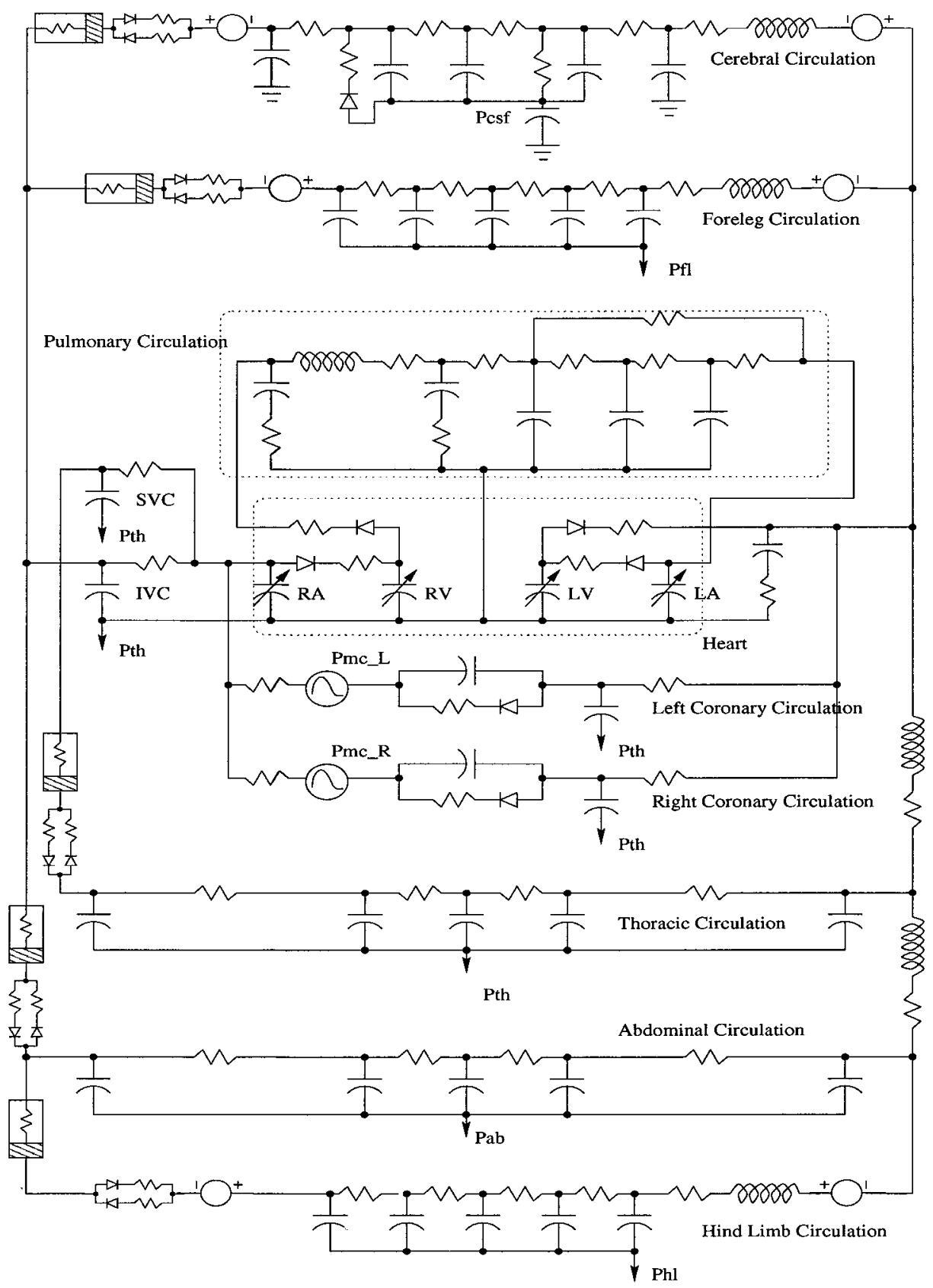

Figure 2.4 The equivalent fluid-mechanical diagram of ovine cardiovascular system. The pulmonary circulation is modified from [2]. The systemic circulation is divided into cerebral, coronary, thoracic, abdominal, foreleg, and hind-limb circulations. 


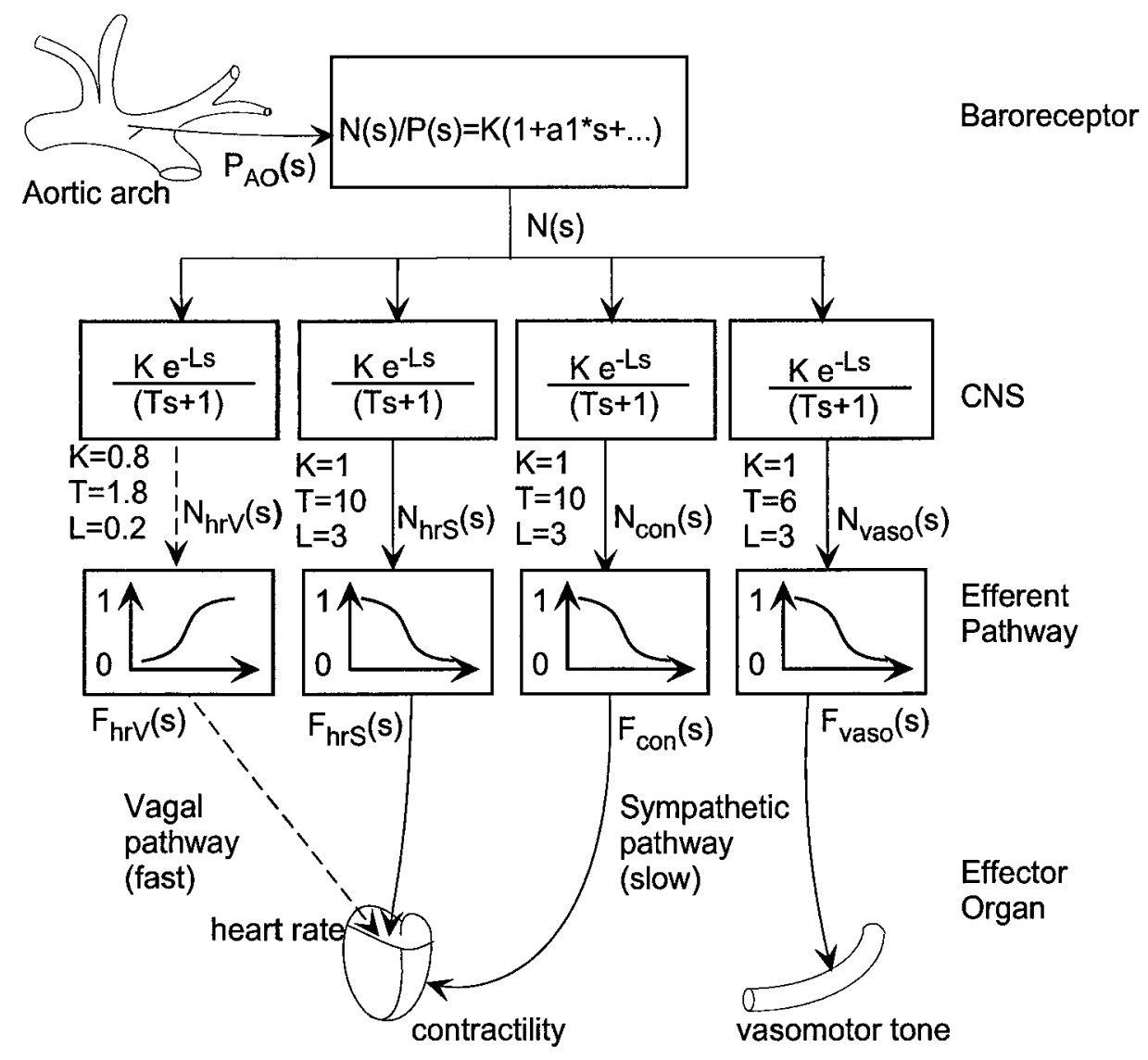

Figure 2.5 The baro-reflex control system(from [3])

\section{Heart Rate}

Heart rate is subject to sympathetic and parasympathetic modulation. We utilize the relation between heart rate and normalized vagal and sympathetic efferent frequencies proposed in studies from Sunagawa et al. [16]:

$$
H R=h_{1}+h_{2} * F_{h r S}-h_{3} * F_{h r S}^{2}-h_{4} * F_{h r V}
$$




$$
+h_{5} * F_{h r V}^{2}-h_{6} * F_{h r V} * F_{h r S}
$$

where $F_{h r V}$ and $F_{h r S}$ are the normalized vagal and sympathetic efferent frequencies, respectively, which are defined in [3]. Parameters $h_{1}$ to $h_{6}$ are constants.

\section{Heart Muscle Contractility}

The autonomic control of the myocardial contractility of the right and left hearts has been discussed previously in $\mathrm{Lu}$ et al [3]. Contractility is modulated by the adjustments of sympathetic tone, which modifies the amplitude and time courses of the elastance curves of the ventricles (Fig. 2.6).

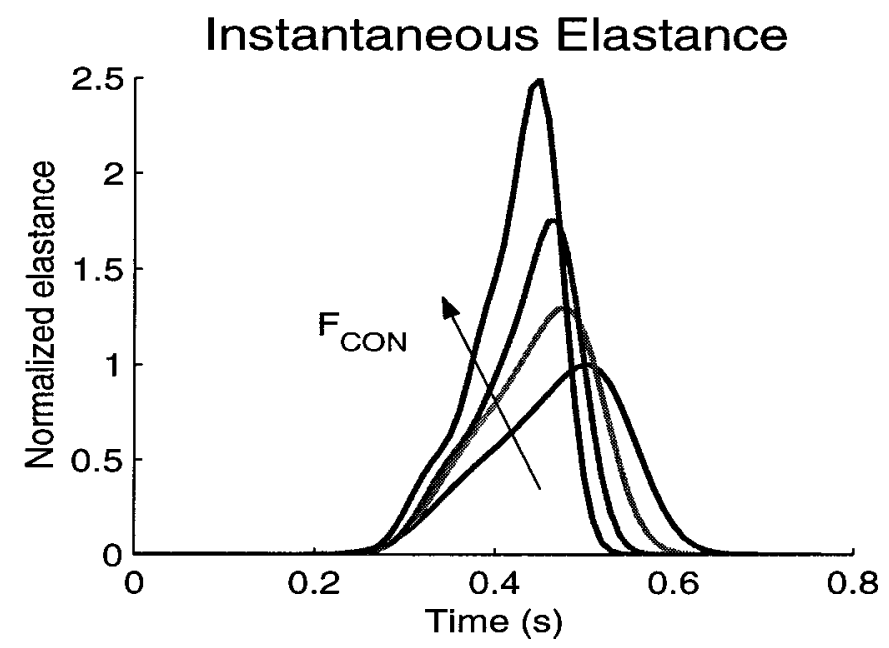

Figure 2.6 Neural modulation of instantaneous ventricular elastance. Shown in the figure are four different levels of contractility corresponding to different sympathetic efferent frequencies $F_{C O N}$. (From Lu et al [3]) 


\section{Vasomotor Control}

Systemic arterioles are the major resistance vessels in the circulation. Lined with smooth muscles, arterioles exhibits a resistance that is modulated over a wide range by sympathetic nervous activity. This process is modeled using a scheme developed by Lu et al. [3], which is based on the experimental work on length-tension relationships of vascular smooth muscle in isolated arterioles by Gore and Davis [18]. Two P-V curves for the arteriole (fully activated and fully relaxed) are shown in Fig. 2.7, and the corresponding $\mathrm{P}-\mathrm{V}$ relations are given in following equations.

$$
\begin{aligned}
P_{a r}^{a}\left(V_{a r}\right)= & K_{a} * \log \left(\frac{\left(V_{a r}-V_{a r, 0}\right)}{D_{0}}+1\right) \\
P_{a r}^{p}\left(V_{a r}\right)= & K_{p 1} * e^{\tau_{p} *\left(V_{a r}-V_{a r, d}\right)} \\
& +K_{p 2} *\left(V_{a r}-V_{a r, d}\right)^{2}
\end{aligned}
$$

$P_{a r}^{a}\left(V_{a r}\right)$ is the $\mathrm{P}-\mathrm{V}$ relation of the arterioles when smooth muscle is in a state of full contraction, whereas $P_{a r}^{p}\left(V_{a r}\right)$ describes the arterioles with sympathetic stimulation abolished. The normalized sympathetic efferent index $F_{V A S O}[3]$ serves as a scaling factor for the transitions between the two extremes. The neural modulation of the axial arterioles resistance is also mathematically described as a function of $F_{V A S O}$

and lumen volume $V_{a r}, R_{a r}=K_{r} * e^{4 * F_{V A S O}}+K_{r} *\left(\frac{V_{a r, M a x}}{V_{a r}}\right)^{2}$, where $K_{r}$ is a scaling factor. 


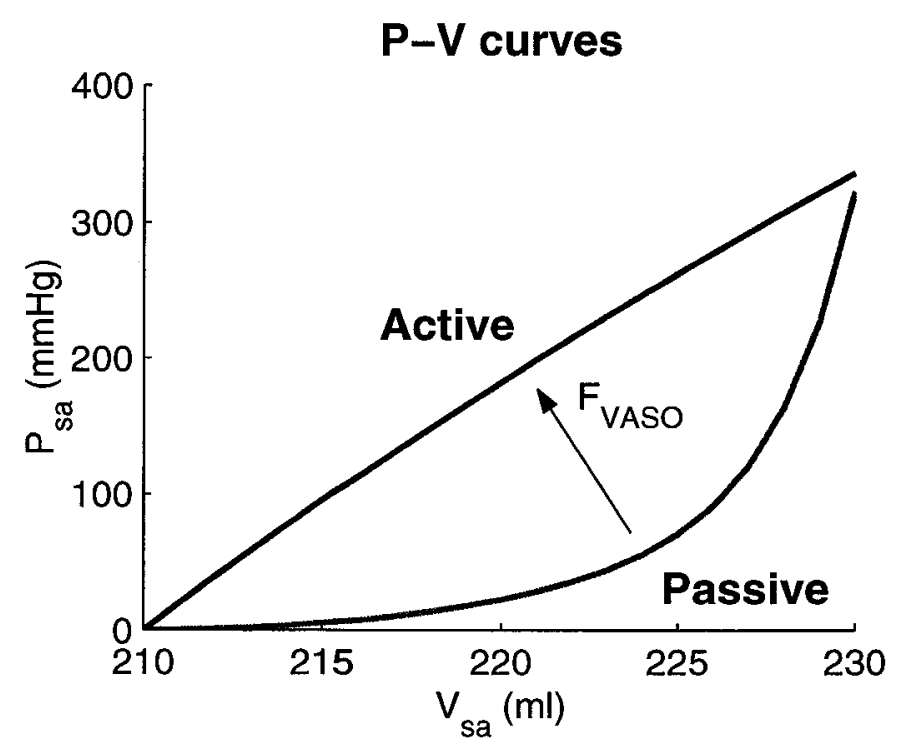

Figure 2.7 An illustration of typical P-V relation curves for the systemic arterioles. The active curve describes the P-V relation of the fully contracted arterioles, while the passive curve describes the nerve-abolished arterioles. A scaling factor mediates the actual instantaneous P-V relation between the two extremes. (From Lu et al [3])

\section{Venomotor Control}

Like arterioles, smaller veins can also contract in response to sympathetic stimulation (venoconstriction). Small compliant veins accommodate almost half the total blood volume, so even a minor constriction can result in a significant increase in venous return to the heart. The venomotor mechanism implemented for venous constriction is similar to that used for the arterioles. And the same normalized sympathetic efferent index $F_{V A S O}$ is used to mediate the venous tone over the full range between the fully activated state and the relaxed (rest) state. 


\subsection{Simulation Result}

\subsubsection{Baseline Model Output}

Baseline model output is produced by tuning the model to simulate data from a healthy, open-chest, paced, mechanically ventilated sheep in the supine position. Figure 2.8 shows plots of model-generated left and right-side pressures in the four heart chambers, the central aortic pressure, pulmonary artery pressure, and flow in central aorta, pulmonary artery, and coronary artery. Table 2.1 summarizes the hemodynamic indices of the simulation.

A

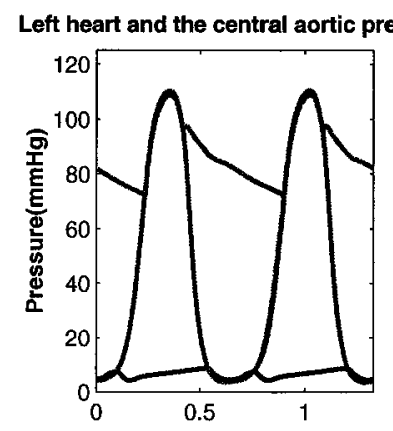

c

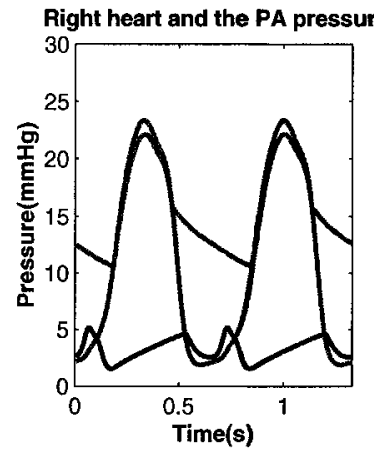

P-V Relation of LV
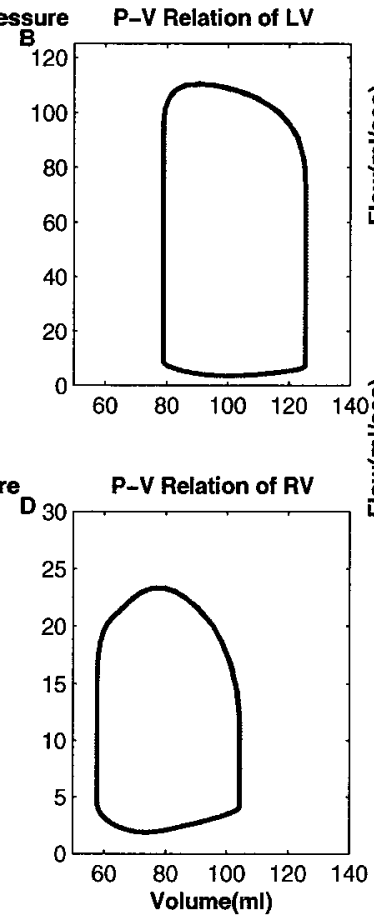
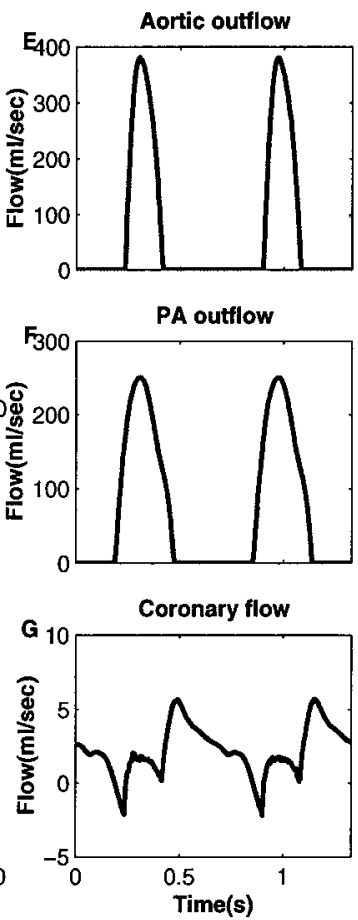

Figure 2.8 Baseline model output 
Table 2.1 Hemodynamic indices

\begin{tabular}{|c||c|c|}
\hline HR & 90 (paced) & min $^{-1}$ \\
\hline SV & 46.4 & $m l$ \\
\hline CO & 4.0 & $l / m i n$ \\
\hline AP & $88.9(109.1 / 72.4)$ & $m m H g$ \\
\hline PP & 36.7 & $m m H g$ \\
\hline LVEP & 27.3 & percent \\
\hline RVEP & 42.7 & percent \\
\hline PAP & $15.6(22.2 / 10.4)$ & $m m H g$ \\
\hline PVP & 7.9 & $m m H g$ \\
\hline
\end{tabular}

\subsubsection{Adaptation to Orthostatic Stress}

With the baseline state established, we consider the transient changes in pressure and volume, when the virtual sheep changes from the prone sitting to a standing position. The left side of Fig. 2.9 shows changes in heart rate variation, peripheral resistance, and MAP (Mean Arterial Pressure) measured at the aorta, in response to a sudden change in posture (sitting to standing). Figure $2.9 \mathrm{E}$ and $\mathrm{F}$ show the changes in the model-generated instantaneous P-V relations for the left and right ventricles during this maneuver. Arrows in the diagrams indicate the direction of the adaptation. Specifically, upon standing the MAP measured at aorta drops dramatically, 
which activates the baroreflex control system. Heart rate is immediately increased, due to a decreased parasympathetic neural discharge frequency and an increased sympathetic frequency. Cardiac output displays an overshoot above the original level immediately after standing up, and then it drops rapidly to a level below that of the supine position. Peripheral resistance is also increased, although more gradually, due to increased sympathetically mediated arteriolar and venous constriction. As a result, MAP increases from the nadir of the response. After a few oscillations (about 15 heart cycles), MAP, heart rate, cardiac output, and peripheral resistance reach a new steady-state. Figure 2.9 also shows that the rising edge of the heart rate variation is quicker than that of the peripheral resistance, reflecting the greater reaction speed of the parasympathetic compared with the sympathetic nervous system.

Although increases in heart rate and peripheral resistance help compensate the reduction in MAP upon standing, venous return to the heart and the cardiac output are reduced. This is evident in panels $\mathrm{E}$ and $\mathrm{F}$ of Fig. 2.9, where the P-V loops for both ventricles shift toward the lower left corner of the diagram, and operate with a decreased stroke volume and pulse pressure.

\subsubsection{Intra Pulmonary Artery Balloon Inflation}

For this maneuver we assume that a Swan-Ganz catheter with balloon is placed in the proximal pulmonary artery $(\mathrm{PA})$. With balloon inflation, there is a sudden increase in pulmonary input impedance [20] measured at the entrance to the PA. Figure 2.10 A 
A

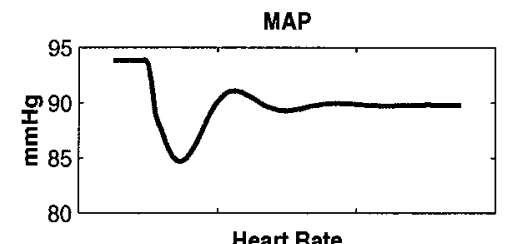

B

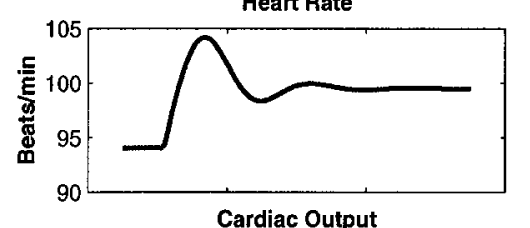

C

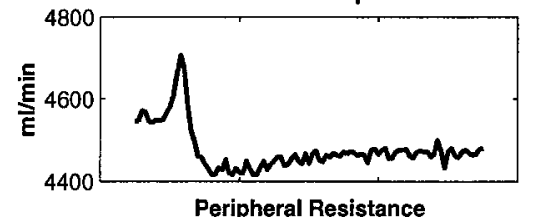

D

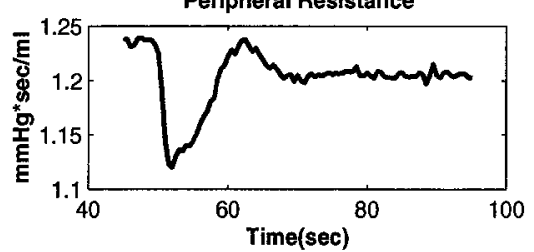

E

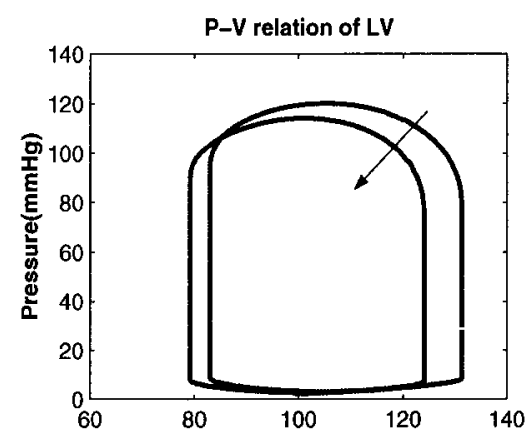

F

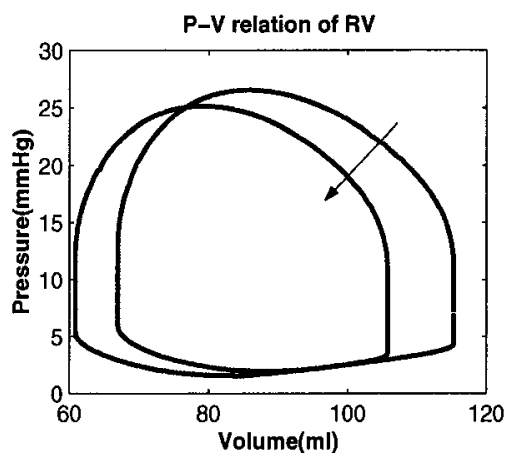

Figure 2.9 Hemodynamic adaptation during standing up. The left four panels show the variation of the MAP (Mean Aortic Pressure), heart rate, cardiac output, and peripheral resistance. The right two panel show the adaptation of the heart pumps using P-V relation loops.

and B show the time courses of PA pressure and outflow, respectively. In response to balloon inflation on the 30th second, PA pressure increases and PA outflow decreases, both to a new steady state. Panels $\mathrm{C}$ and $\mathrm{D}$ show the input impedance (amplitude and phase) under control condition and balloon inflation. The arrow indicates the direction of change from the first steady-state to the second. Figures $2.10 \mathrm{E}$ and F show the P-V loops of both ventricles, whereas Fig. $2.10 \mathrm{G}$ and $\mathrm{H}$ show the muscle work (MW) and the energy efficiency index (EEI) for both ventricles. 
As the arrows show, the P-V loop of the RV shifts to the upper right corner after the balloon inflation, whereas the LV P-V loop shifts to lower left corner. Inflating the balloon increases RV afterload and reduces right ventricular outflow, resulting in a higher RV volume and an elevated RV pressure. Our interactive ventricular model ([1], [3]) shows that the elevated RV pressure expands the RV lumen toward the LV, which further increases the RV volume and decreases the LV volume. The increased total pulmonary resistance reduces pulmonary venous pressure, reducing LVED as well. This shown in Fig. $2.10 \mathrm{~F}$ where the LV P-V loop shifts to the lower left corner. In response to balloon inflation, the stroke volumes of both ventricles are reduced, although for different reasons. For the RV it is due to an increased afterload, whereas for the LV it is due to a decreased preload. We compute the MW and the EEI of both ventricles based on the respective P-V loops. Figure $2.10 \mathrm{G}$ and $\mathrm{H}$ show that following the balloon inflation the RV does more external work (RVMW), while the LV does less than before inflation (LVMW). The efficiency of both ventricles (RVEEI and LVEEI) drops, implying that both ventricles work optimally with their normal preloads and afterloads in terms of the efficiency of converting chemical energy to external work. The definitions and computation methodology for MW and EEI are given in the appendix. 


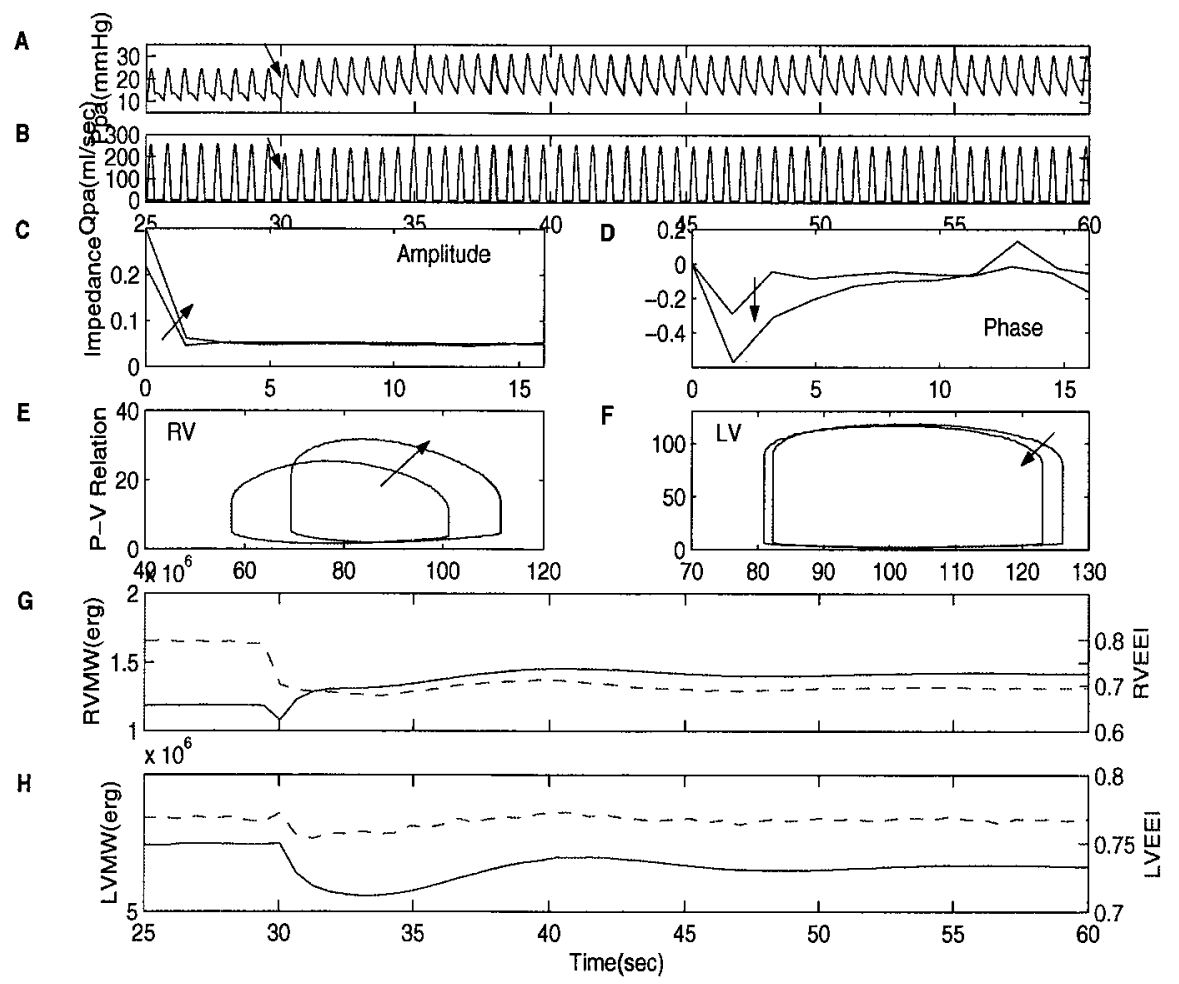

Figure 2.10 Virtual experiment of the Intra-PA Balloon Inflation. From top to bottom, the PA pressure, the PA outflow, the PA impedances of two steady states, P-V loops of both ventricles, RVMW, RVEEI, LVMW, LVEEI are shown.

\subsection{Discussion}

We have presented a mathematical model of the ovine cardiovascular system. The model produces baseline output that correlates well with typical hemodynamic data from an anesthetized open-chest sheep. Calculated hemodynamic indices, such as $\mathrm{CO}, \mathrm{SV}$, and EF, likewise correlate with published data([30], [31], [32], [33], [34], $[36])$. 


\subsubsection{Simulation of Orthostatic Stress}

As noted previously, this model is capable of simulating blood volume redistribution among major systemic circulations upon perturbation. In the orthostatic stress challenge experiment, the aortic volume is partially re-distributed to the limb circulations when the sheep suddenly stands up. As the result of the volume shift, there is a dramatic initial decrease in MAP measured at the aorta (Fig. 2.9) and an increase in limb arterial pressure. Similarly, the blood pressure in the vena cava decreases as the blood is partially redistributed to the limbs, while the limb venous pressure

increases accordingly (Fig. 2.11). The model simulates the compensatory response to this blood volume shift via the neural venomotor control, the volume-dependent resistance of the large veins, and the pressure-operated venous valves. When the sheep stands up, venomotor tone is increased as the rapid reduction in arterial pressure triggers the baroreflex control. Small veins in the limb circulations contract and return large quantities of pooled blood to the large veins. As the volume of the large veins decreases because of blood shift to the lower limbs, their resistance increases, thus slowing down the process of relocation. Furthermore, the pressure-operated venous valves, which display a high resistance when the pressure upstream is less than the pressure downstream, help to stop the volume shift when the pressure in the vena cava is low. This mechanism is crucial in maintaining a minimum venous return under conditions of orthostatic stress. 

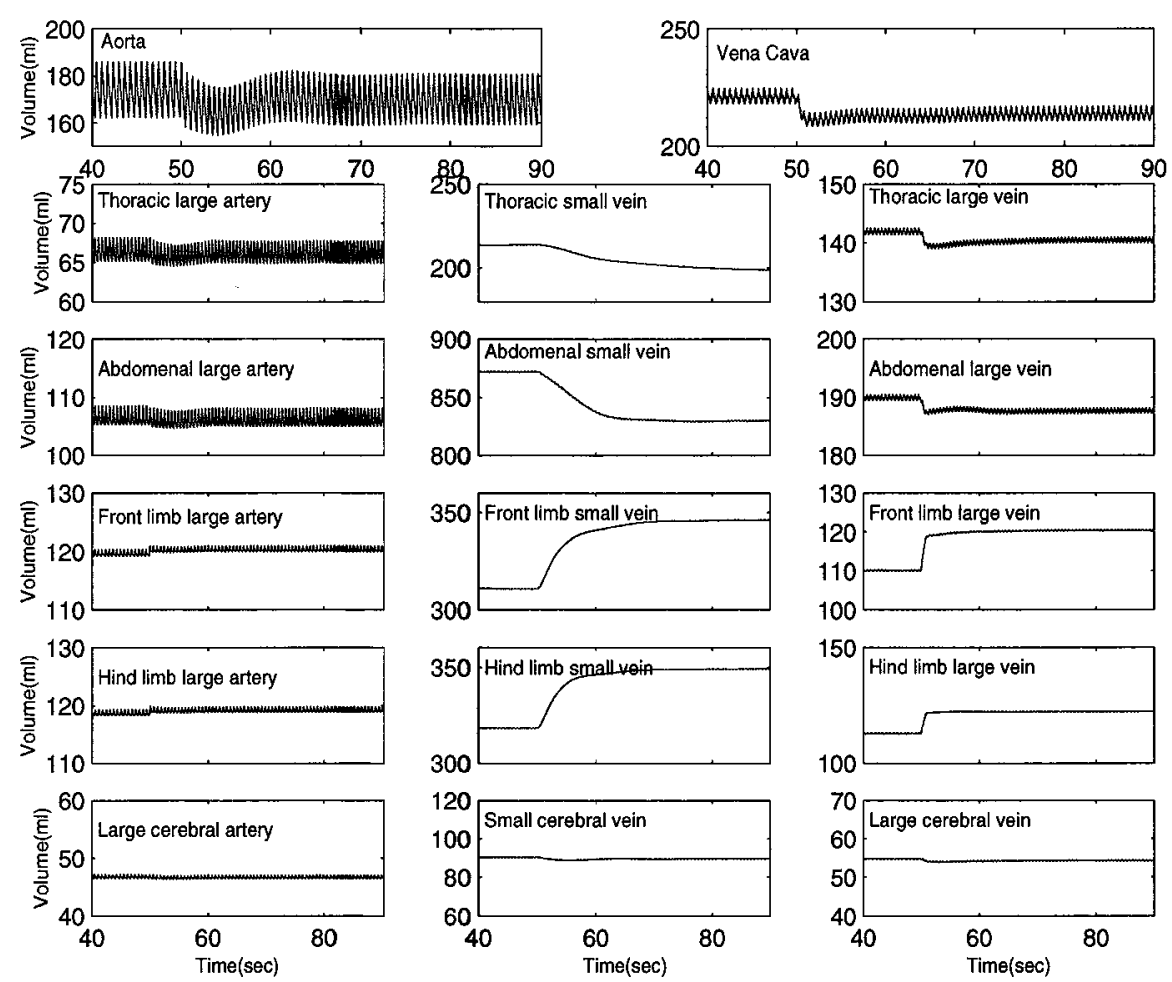

Figure 2.11 Volume redistribution under orthostatic stress challenge

\subsubsection{Rapid Increase in RV Afterload}

One of the fundamental purposes of cardiovascular modeling is to describe the pump-load interaction between heart and vasculature. The interaction between LV and the systemic circulation is evident in the response to orthostatic stress (Fig. 2.9), which shows that reduced peripheral resistance decreases cardiac output and increases heart rate. On the other hand, the balloon inflation experiment demonstrates the interaction between RV and the pulmonary circulation. As Fig. 2.12 shows, increased pulmonary load decreases cardiac output and increases heart rate. 
Since heart rate is largely determined by the systemic arterial pressure rather than pulmonary arterial pressure, the heart rate increase is much slower than that shown for the orthostatic challenge (Fig. 2.9). An important part of this response is the di-
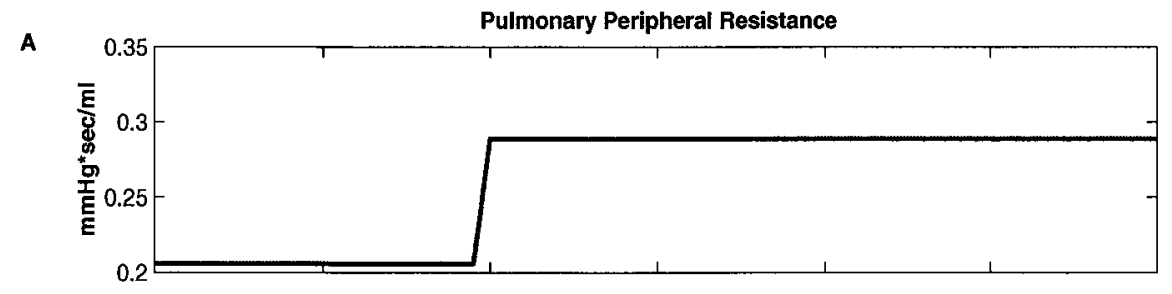

B

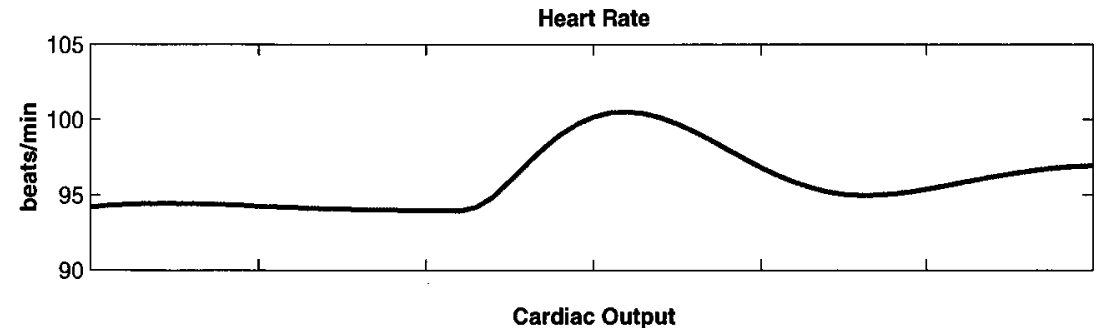

c

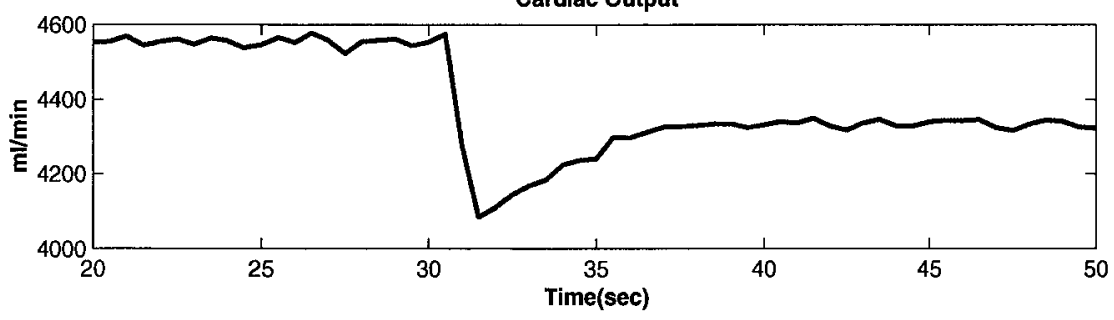

Figure 2.12 The interaction between RV and pulmonary circulation

rect (parallel) ventricular interaction between the $R V$ and $L V$, as well as, the indirect (series) interaction that takes place between the pulmonary and systemic circulations. These interaction terms are defined in [2]. To show the effect of direct ventricular interaction, we make the septum "rigid" [1], and run the balloon inflation experiment for comparison. With a rigid septum, direct parallel interaction between pumps is 
eliminated. The only interaction between ventricles is the indirect (series) type, that is, via the circulatory network. Figure $2.13 \mathrm{C}$ shows that for the rigid septum, the increase in RVED and RVES is less than in the control case where direct ventricular interaction is included (Fig. 2.13 A). Similarly, Fig. 2.13 D shows that the decrease in LVED and LVES is also less, compared with control (Fig. 2.13 B).

A

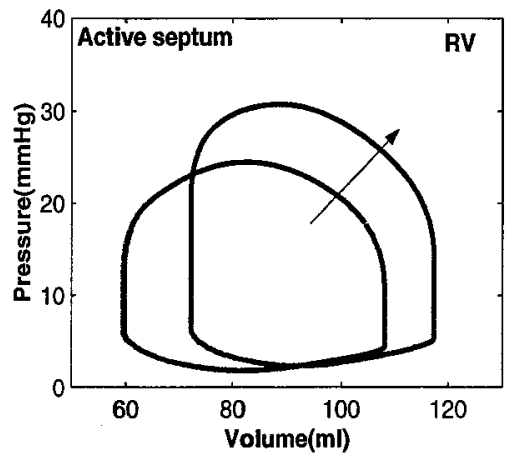

c

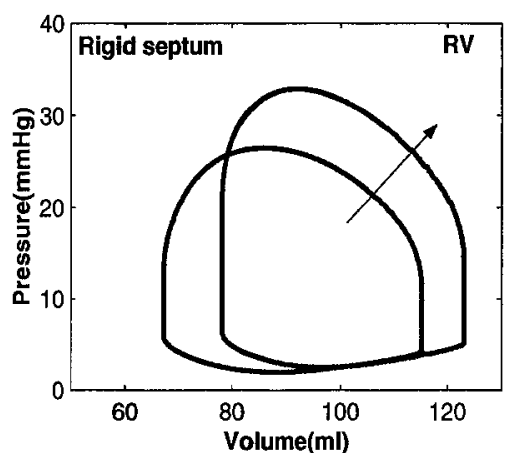

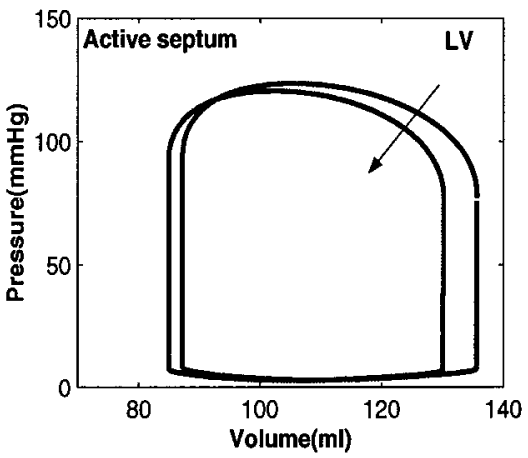

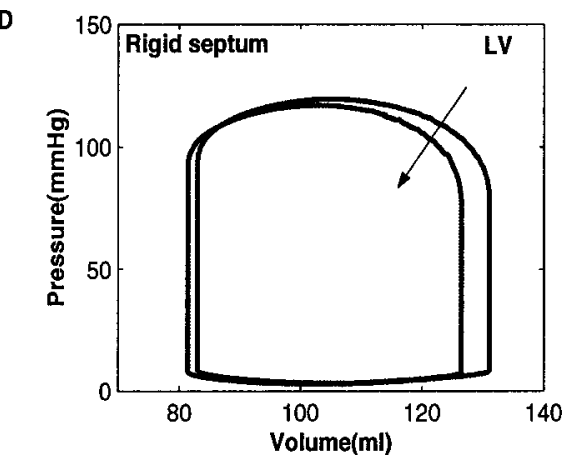

Figure 2.13 The interaction between RV and LV 


\section{Chapter 3 PAL Modeling and Virtual Testing}

The PAL (Paracorporeal Artificial Lung) has been developed as a temporary pulmonary assist device. Here we examine the hemodynamic effects of its implantation in sheep, by developing a lumped parameter model of the PAL and connecting it to our whole body circulatory model of the sheep. By varying parameter values of the PAL device model, we can also test the putative effectiveness of different designs.

\subsection{Development of PAL Device Model}

Figure 3.1 shows surgical attachment of the PAL device to the pulmonary circulation, whereas Fig. 3.2 shows the hydraulic equivalent circuit model of the pulmonary circulation with the model of the PAL device embedded. The dashed block shown in Fig. 3.2 highlights the implantation and includes hydraulic equivalent circuit of model of the inlet and outlet grafts, whereas the interior dotted block shows the model of the device itself.

Table 3.1 contains baseline values estimated for the design parameters of both the PAL device and the grafts. The compliance of the inlet and outlet tubes are estimated according to the following formula for a thick elastic cylinder:

$$
C=\frac{3 \pi r^{3}}{2 h E} l
$$

where $r$ is the cylinder radius, $E$ the Young's modulus of the wall material, $h$ the 


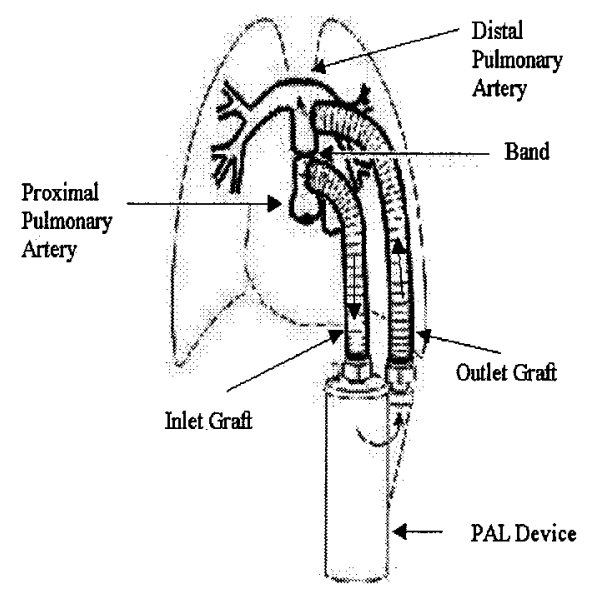

Figure 3.1 The PAL attachment

wall thickness, and $l$ the length of the segment. We choose the baseline design as 15 $\mathrm{cm}$ long, $1 \mathrm{~cm}$ in diameter, $0.1 \mathrm{~cm}$ in wall thickness, and teflon as the graft material $\left(E=5.8 * 10^{8} \mathrm{~N} / \mathrm{m}^{2}\right)$. The inertance of the grafts is calculated according to

$$
L=\frac{4 \rho l}{\pi r^{2}}
$$

where $\rho$ is the blood density (normally, $1.504 \mathrm{~g} / \mathrm{cm}_{3}$ ), $l$ the length of the tube, and $r$ the radius. We calculate the resistance of the graft to the blood flow according to the formula for Poiseuille flow:

$$
R=\frac{8 \eta l}{\pi r^{4}}
$$

where $\eta$ is the viscosity. 


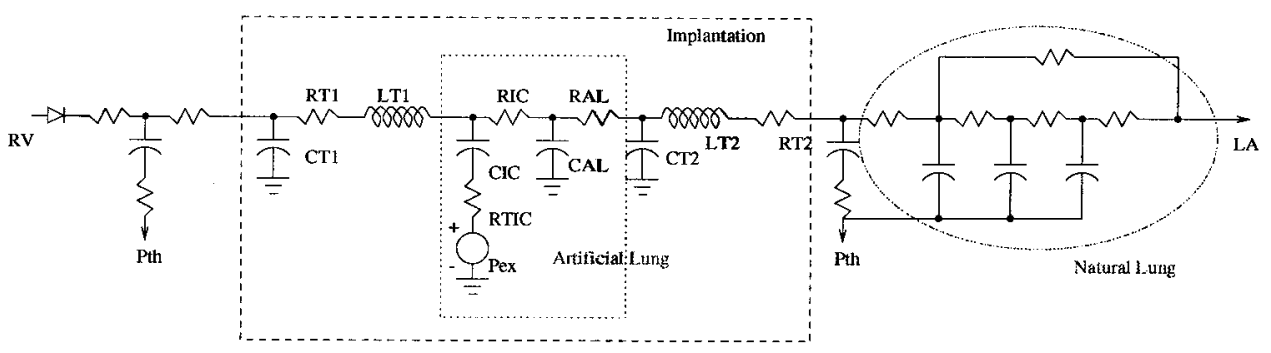

Figure 3.2 Hydraulic equivalent model of the PAL connected in series with the proximal and distal pulmonary artery.

\subsection{Hemodynamics after Implantation of Baseline-Design PAL}

Using these nominal parameter values, a baseline output of the integrated model containing the PAL model is generated. Figure 3.3 A shows right ventricular pressure $\left(P_{R V}\right)$, pulmonary arterial pressure $\left(P_{P A}\right)$, and right atrium pressure $\left(P_{R A}\right)$. Figure $3.3 \mathrm{~B}$ shows the pressure in the artificial lung chamber $\left(P_{A L}\right)$, whereas Figures 3.3 $\mathrm{C}$ and $\mathrm{D}$ show the blood flow through proximal pulmonary artery $\left(Q_{P A}\right)$ and the artificial lung $\left(Q_{A L}\right)$, respectively. Figures $3.3 \mathrm{E}$ and $\mathrm{F}$ show the P-V relation curve of the right ventricle (RV) and the left ventricle $(\mathrm{LV})$, respectively. For comparison, we also plot the P-V curves of a normal control sheep without the PAL device in dotted lines. Obviously, PAL implantation elevates the RV pressure, increases its end-diastolic volume, but decreases its stroke volume (the width of the P-V loop is narrowed). In the case of left ventricle, due to a decrease in RV stroke volume, thus a 
Table 3.1 Baseline parameter values of PAL device and the grafts

\begin{tabular}{|c|l|l|l|}
\hline Parameter & Description & Value & Unit \\
\hline CT1 & Inlet Tube Compliance & 0.031 & $\mathrm{ml} / \mathrm{mmHg}$ \\
\hline RT1 & Inlet Tube Resistance & 0.00115 & $\mathrm{mmHg} * \mathrm{sec} / \mathrm{ml}$ \\
\hline LT1 & Inlet Tube inertance & 0.0143 & $\mathrm{mmHg} * \mathrm{sec} / \mathrm{ml}$ \\
\hline CIC & Inflow Chamber Compliance & 1.0 & $\mathrm{ml} / \mathrm{mmHg}$ \\
\hline RIC & Axial Inflow Chamber Resistance & 0.001 & $\mathrm{mmHg} * \mathrm{sec} / \mathrm{ml}$ \\
\hline RTIC & Transmural Inflow Chamber Resistance & 0.001 & $\mathrm{mmHg} * \mathrm{sec} / \mathrm{ml}$ \\
\hline CAL & Compliance of Artificial Lung Chamber & 1.0 & $\mathrm{ml} / \mathrm{mmHg}$ \\
\hline RAL & Resistance of Artificial Lung Chamber & 0.078 & $\mathrm{mmHg} * \mathrm{sec} / \mathrm{ml}$ \\
\hline CT2 & Outlet Tube Compliance & 0.031 & $\mathrm{ml} / \mathrm{mmHg}$ \\
\hline RT2 & Outlet Tube Resistance & 0.00115 & $\mathrm{mmHg} * \mathrm{sec} / \mathrm{ml}$ \\
\hline LT2 & Outlet Tube inertance & 0.0143 & $\mathrm{mmHg} * \mathrm{sec} / \mathrm{ml}$ \\
\hline
\end{tabular}

decrease in LV preload, and the fact that increased RV pressure expands the septum towards the left side, its end-diastolic volume is decreased. As a result, its systolic pressure is also decreased.

Pulmonary arteries, like their systemic counterparts, act both as blood conduits and as cushions to smooth flow pulsations imposed by contracting heart ([21], [22]). And the efficiency of cushioning function depend on geometric and viscoelastic prop- 
A

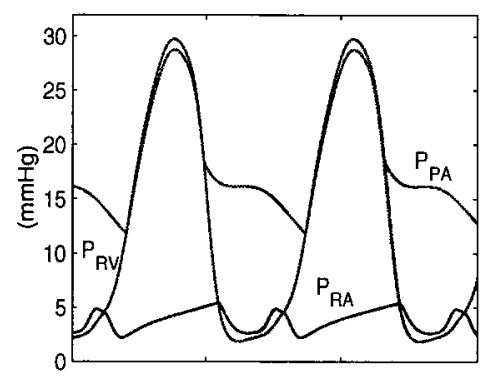

$\mathbf{B}$

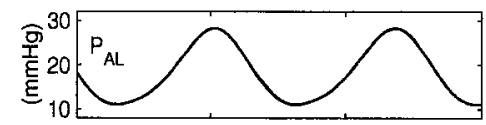

c

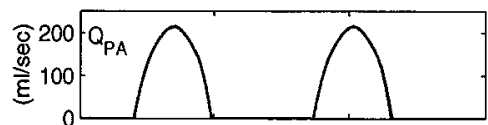

D

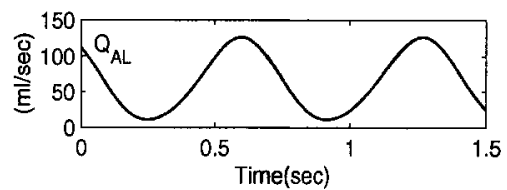

E
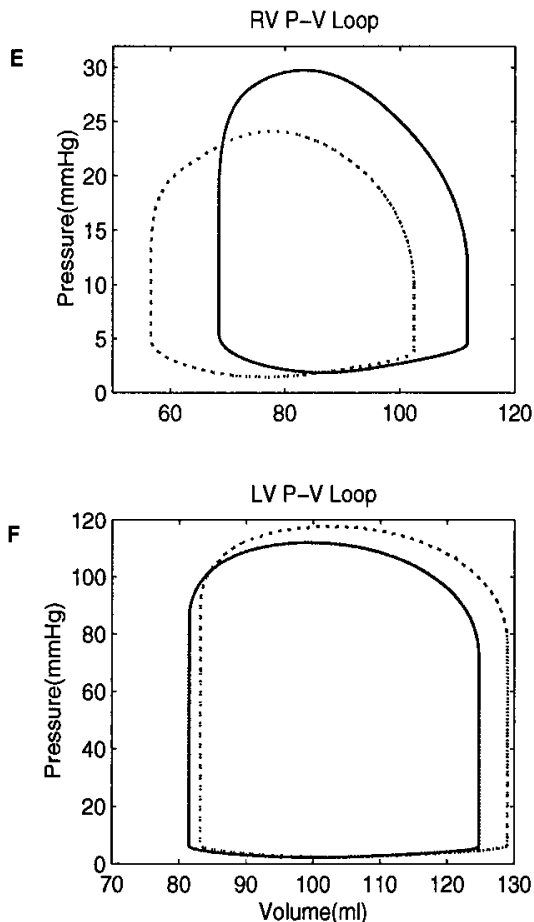

Figure 3.3 Hemodynamics after Implantation of Baseline-Design PAL.

erties of the circulatory load described in terms of compliance [22]. Attachment of the relatively stiff inlet graft to the proximal pulmonary artery reduces the effective pulmonary circulatory compliance substantially. The reduced effective compliance results in weakened cushioning function of the pulmonary circulation, which in turn results in increased pulmonary artery pulse pressure (PAPP) and systolic right ventricular pressure (SRVP)(Table 3.2), and causes right heart to do more work to maintain a normal cardiac output. 
Table 3.2 Hemodynamic changes induced by PAL implantation

\begin{tabular}{|c||c|c|c|}
\hline & Baseline & PAL implanted & Unit \\
\hline PAPP & 9.1 & 16.5 & $m m H g$ \\
\hline SRVP & 22.4 & 27.7 & $m m H g$ \\
\hline RVMW & 1.06 & 1.24 & $10^{6} \mathrm{erg}$ \\
\hline RVEEI & 0.814 & 0.696 & \\
\hline CO & 4.0 & 3.9 & $l / \mathrm{min}$ \\
\hline
\end{tabular}

\subsection{Sensitivity of PAL Design Parameters}

Different designs of the PAL device is tested on the whole body model, by varying the compliance (CT1) and the inertance of the inlet tube (LT1), and the compliance of the inflow chamber (CIC) and the resistance (RAL) of the PAL device.

\subsubsection{CT1}

The baseline value for CT1 $(0.031 \mathrm{ml} / \mathrm{mmHg})$ is small and corresponds to a rigid tube. We simulate more compliant tubes by changing CT1 to $0.5 \mathrm{ml} / \mathrm{mmHg}$ and 1.0 $\mathrm{ml} / \mathrm{mmHg}$. Table 3.3 shows that increasing CT1 lowers the systolic right ventricular pressure (SRVP) and right ventricular muscle work (RVMW). It also increases the energy efficiency of the right ventricle (RVEEI). Cardiac output (CO) is influenced minimally as the tube is made more compliant, indicating that whereas SRVP drops 
significantly, the mean pulmonary arterial pressure only drops a little. Increased CT1 also decreases the pulsation of $P_{A L}$ and $Q_{A L}$, whereas their mean values change little.

Table 3.3 The effects of inlet tube compliance (CT1)

\begin{tabular}{|l|c|c|c|c|c|c|}
\hline CT1 & RVMW & RVEEI & CO & SRVP & $P_{A L}(\mathrm{M} /(\mathrm{S} / \mathrm{D}))$ & $Q_{A L}(\mathrm{M} /(\mathrm{S} / \mathrm{D}))$ \\
\hline $0.031 *$ & 1.35 & 0.676 & 3.90 & 29.8 & $18.7(28.3 / 11.0)$ & $65.1(126.9 / 11.5)$ \\
\hline 0.5 & 1.24 & 0.684 & 3.88 & 28.0 & $18.6(26.9 / 11.8)$ & $64.7(118.4 / 17.1)$ \\
\hline 1.0 & 1.17 & 0.696 & 3.87 & 26.4 & $18.5(25.7 / 12.6)$ & $64.6(110.5 / 23.3)$ \\
\hline
\end{tabular}

* baseline, RVMW is in $10^{6} \mathrm{erg}$, CO in $l / \mathrm{min}$, SRVP and $P_{A L}$ in $m m H g$, and $Q_{A L}$ in $m l / s e c$

\subsubsection{LT1}

If we assume that the radius of the inlet graft is kept constant, the inertance of the tube depends on its length. The longer the tube, the larger inertance it imparts to the blood column it contains. Table 3.4 shows that an increased inertance places an additional load on the right ventricle, causing right ventricular systolic pressure (SRVP) and muscle work (RVMW) increase, and efficiency (RVEEI) to decrease. The greater inertance also increases the pulsility of $P_{A L}$ and $Q_{A L}$.

\subsubsection{RAL}

Table 3.5 shows the effect of a change in PAL resistance (RAL) on heart performance and PAL device hemodynamics. A high PAL resistance of results in a smaller 
Table 3.4 The effects of inlet tube inertance (LT1)

\begin{tabular}{|l|c|c|c|c|c|c|}
\hline LT1 & RVMW & RVEEI & C0 & SRVP & $P_{A L}(\mathrm{M} /(\mathrm{S} / \mathrm{D}))$ & $Q_{A L}(\mathrm{M} /(\mathrm{S} / \mathrm{D}))$ \\
\hline $0.0096(10 \mathrm{~cm})$ & 1.33 & 0.691 & 3.93 & 29.1 & $18.8(28.6 / 11.7)$ & $65.5(126.1 / 17.5)$ \\
\hline $0.0143(15 \mathrm{~cm}) *$ & 1.35 & 0.676 & 3.90 & 29.8 & $18.7(28.3 / 11.0)$ & $65.1(126.9 / 11.5)$ \\
\hline $0.0191(20 \mathrm{~cm})$ & 1.38 & 0.660 & 3.87 & 30.9 & $18.5(28.2 / 10.5)$ & $64.6(126.7 / 8.2)$ \\
\hline
\end{tabular}

*baseline, RVMW is in $10^{6} \mathrm{erg}, \mathrm{CO}$ in $l / \mathrm{min}$, SRVP and $P_{A L}$ in $m m H g$, and $Q_{A L}$ in

$$
m l / s e c
$$

cardiac output (CO), and a higher systolic right ventricular pressure (SRVP). A more resistive PAL device makes the right ventricle do more work (RVMW) at a lower level of energy utilization efficiency. Reduced cardiac output in turn makes left ventricle output less work, and the left ventricular energy efficiency remains largely the same. The variation of RAL also has significant influence on the pulsation of $Q_{A L}$. A low RAL results in a dramatic increase in the pulse flow of $Q_{A L}$, as shown in Table 3.5.

\subsubsection{CIC}

The effects of the different designs of the inflow chamber on the right ventricular muscle work (RVMW), the systolic right ventricular pressure (SRVP), and the pulse flow through the PAL device (PQAL) are shown in Fig. 3.4. At low CAL, which means rather stiff inflow chamber, the SRVP, and thus the oxygen consumption, is 
Table 3.5 The effects of PAL resistance (RAL)

\begin{tabular}{|l|c|c|c|c|c|c|}
\hline RAL & RVMW & RVEEI & CO & SRVP & $P_{A L}(\mathrm{M} /(\mathrm{S} / \mathrm{D}))$ & $Q_{A L}(\mathrm{M} /(\mathrm{S} / \mathrm{D}))$ \\
\hline 0.02 & 1.15 & 0.708 & 4.00 & 26.2 & $15.3(23.7 / 7.9)$ & $66.7(140 /-1.3)$ \\
\hline $0.078 *$ & 1.35 & 0.676 & 3.90 & 29.8 & $18.7(28.3 / 11.0)$ & $65.1(126.9 / 11.5)$ \\
\hline 0.15 & 1.54 & 0.634 & 3.78 & 34.1 & $22.6(33.3 / 14.4)$ & $63.0(113.7 / 21.0)$ \\
\hline
\end{tabular}

high. As CAL increases, SRVP decreases to a nadir when CIC equals $1.0 \mathrm{ml} / \mathrm{mmHg}$, and SRVP increases as CAL further increases. RVMW is low when CIC equals $0.2 \mathrm{ml} / \mathrm{mmHg}$, and it displays a small peak when $\mathrm{CIC}$ equals $0.4 \mathrm{ml} / \mathrm{mmHg}$. It then decreases to a nadir when $\mathrm{CIC}$ equals $0.8 \mathrm{ml} / \mathrm{mmHg}$, after which it again rises till the end of the observation. The variation of PQAL generally flows the same pattern of that of RVMW, which displays two minimum in the spectrum of the observation, one when CIC is very low, the other when CIC assumes medium value.

Figures 3.5 shows the influence of the inflow chamber on the pulmonary impedance. We observe that the installation of an inflow chamber reduces impedance and that varying the compliance of the inflow chamber produces significant changes in the waveforms of both amplitude and phase of the impedance. This demonstrates the double value of the inflow chamber in the artificial lung design, which not only re- 

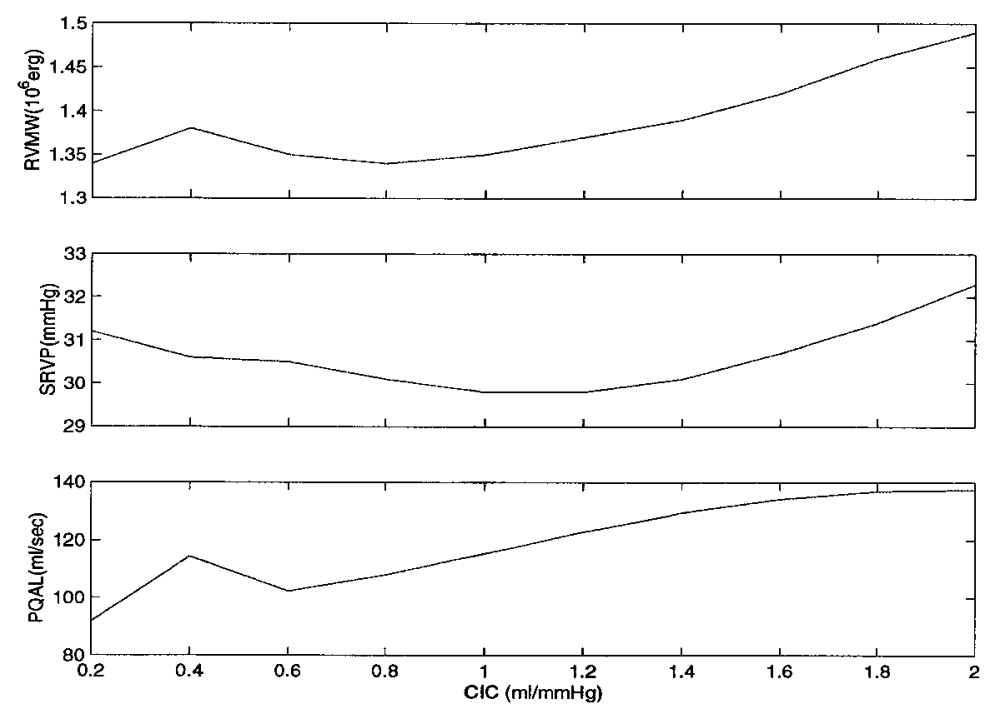

Figure 3.4 The sensitivity of right ventricular muscle work (RVMW), the systolic right ventricular pressure (SRVP), and the pulse flow through the PAL device (PQAL) to the variation of the compliance of the PAL device

duces impedance to the right ventricle, but also provides another vehicle to fine-tune the geometric and viscoelastic characteristics of the whole implantation.

\subsection{Discussion}

We used the model to test the hemodynamic consequences of PAL implantation. Further we investigated the effects of different design parameters on pulmonary hemodynamics and heart energetics. Among various parameters and indices, we concentrate on the examination of RVMW, RVEEI, CO, SRVP (always seen as an index for total oxygen consumption), $P_{A L}$ and $Q_{A L}$. The pulsation of $Q_{A L}$ is especially important, because highly pulsatile flow through the PAL will inevitably affect overall 

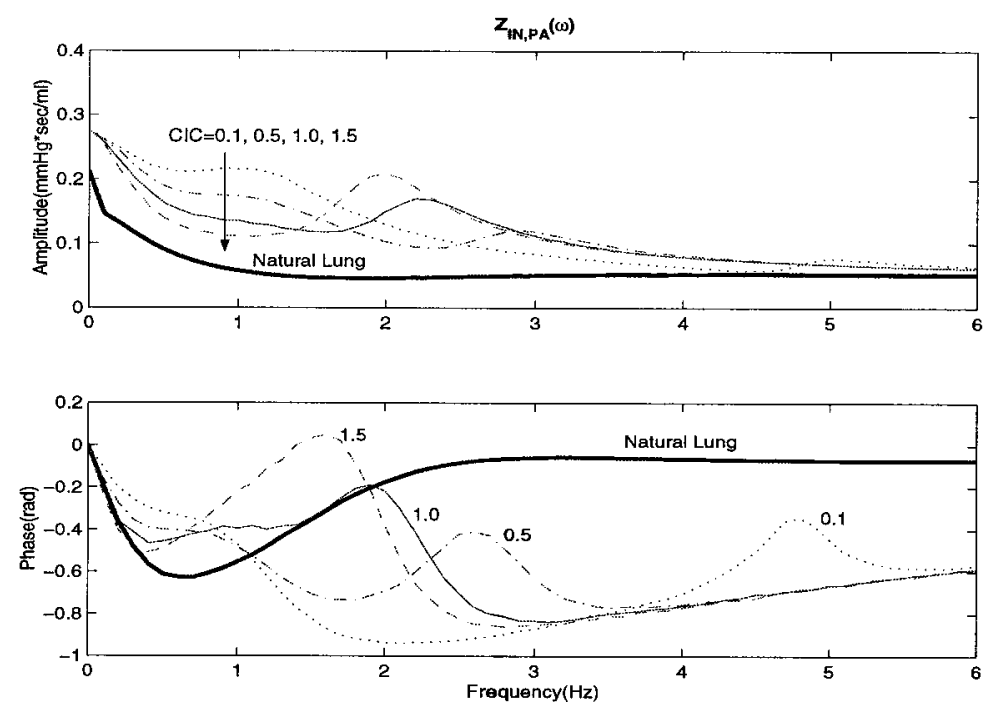

Figure 3.5 The influence of inflow chamber on the pulmonary impedance. The bold solid line represents the impedance when inflow chamber is not installed, the solid line when a baseline-design ( $\mathrm{CIC}=1.0 \mathrm{ml} / \mathrm{mmHg}$ ) inflow chamber is attached, the dotted line when a very stiff one (CIC $=0.1 \mathrm{ml} / \mathrm{mmHg})$ is installed, the dash-dotted line when CIC = $0.5 \mathrm{ml} / \mathrm{mmHg}$, the dashed line when CIC $=1.5 \mathrm{ml} / \mathrm{mmHg}$.

gas exchange ability of the device.

The implantation of PAL makes the pulmonary circulation less compliant to the right ventricle, even with an inflow compliance chamber installed. The consequences are increased pulmonary arterial pulse pressure (PAPP) and systolic right ventricular pressure (SRVP). Since the main reason why the pulmonary circulation becomes less compliant is the rigid inlet and outlet tube which connect the PAL device into the circulation, it is no surprisc that more compliant tubes, especially the inlet tube, will lower systolic right ventricular pressure (SRVP) and the muscle work that RV has to do to maintain a normal cardiac output (CO)(Table 3.3). However, changing CT1 is 
not an easy, feasible choice in practice, for the determining factor of the compliance is the graft material, which has to be rather stiff to be manageable. We do have control over the length of the graft tubes, the resistance of the PAL device, and the compliance (elasticity) of the inflow chamber. These parameters are adjusted for acceptable performance.

\subsubsection{The Length of the Inlet Tube}

As Equations (3.1, 3.2, 3.3) show, the compliance, inertance, and the resistance of the graft are in linear relationship with the length of the inlet tube $(l)$. An increase in $l$ will increase $\mathrm{CT} 1, \mathrm{LT} 1$, and RT1 at the same time. However, compared with natural pulmonary artery, CT1 and RT1 are small, and the linear variation with $l$ does not produce significant changes in hemodynamics. However for LT1, its value is comparable with that of the natural pulmonary artery, hence LT1 variation produces significant changes in right heart energetics and pulmonary hemodynamics (Table 3.4). Table 3.4 implies, the shorter the inlet graft (lower LT1), the lower RVMW, the higher the RV energy efficiency, and the lower pulsatility of $P_{A L}$ and $Q_{A L}$ waveforms.

\subsubsection{The Resistance of the PAL Device (RAL)}

Great efforts have been devoted to reducing the resistance of the PAL device (RAL). However, the conclusion on RAL in this study is not straightforward. On one hand, a lower RAL means lower RV load, so the RV can do less muscle work to 
maintain a normal cardiac output. Table 3.5 shows that a lower RAL also contributes to a higher RV energy efficiency (RVEEI). On the other hand, a lower RAL results in high pulsation of $P_{A L}$ and $Q_{A L}$, which in turn leads to a lower efficiency of gas exchange in the PAL device. We should be aware of this tradeoff when designing the PAL device.

\subsubsection{The Compliance of the Inflow Chamber (CIC)}

Based on different criteria, different "optimal" designs of the inflow chamber can be found. As shown in Fig. 3.4, a stiff inflow chamber, or no inflow chamber at all, is an "optimal design" in terms of reducing pulsatility of flow through the PAL device (PQAL). In terms of reducing total oxygen consumption (reflected by SRVP, Systolic Right Ventricle Pressure), the "optimal design" is an inflow chamber with medium-value compliance $(\mathrm{CIC}=1.0 \mathrm{ml} / \mathrm{mmHg})$. And in terms of right ventricle muscle external work (RVMW), an inflow chamber with a CIC of $0.8 \mathrm{ml} / \mathrm{mmHg}$ is best. In practice, we can choose a CIC such that the resulted impedance best matches the impedance of natural lung. In Fig. 3.5, we observe that the impedance curve associated with the $\mathrm{CIC}$ value of $1.0 \mathrm{ml} / \mathrm{mmHg}$ preserves phase delay between pressure and flow in the frequency range $1.4-1.7 \mathrm{~Hz}$, where most of the hydraulic power provided by the beating heart concentrates. At the same time a value of $1.0 \mathrm{ml} / \mathrm{mmHg}$ for $\mathrm{CIC}$ achieves a low amplitude of impedance in the same frequency range. We can conclude that a value of $1.0 \mathrm{ml} / \mathrm{mmHg}$ for $\mathrm{CIC}$ is the best in terms of 
preserving the natural pulmonary impedance, holding other parameters in the PAL model constant at baseline values. 


\section{Chapter 4 Summary}

This thesis has presented the development of a new whole body cardiovascular model, physiological system analysis based on the model, and practical application of this model in the testing the PAL (Para-corporeal Artificial Lung) device. Original contributions of this study are summarized below.

\subsection{Cardiovascular Modeling}

- A whole-body mathematical model has been developed for the sheep, a popular animal model for human cardiovascular and cardiopulmonary diseases. This model integrates a dynamic heart model with direct ventricular coupling, systemic and pulmonary circulatory model, and a short-term baroreflex control model. The identified model produces realistic, continuous hemodynamic waveforms around the circulatory loop, as well as hemodynamic indices such as cardiac output that conform to published data.

- A distributed approach is adopted in describing the systemic circulation, which is divided into cerebral, foreleg, coronary, thoracic, abdominal, and hind leg circulations. This approach enables study of volume redistribution and hemodynamic changes during postural change.

- A lumped parameter model is developed for the PAL device and its connect- 
ing grafts. Furthermore, relationships between model parameters and practical design parameters such as length of the graft are established. This enables simulations that explore hemodynamic consequences of different designs of the PAL device and the modes of surgical attachment.

\subsection{Physiological Analysis}

- The integrated model is applied to the study of physiological adaptation to orthostatic stress. Computational simulation predicts hemodynamic consequences after the virtual subject assumes an upright position and demonstrates the partial restoration of arterial pressure by baroreflex control mechanism.

- The virtual experiment of intra pulmonary artery balloon inflation demonstrates the ability of this model to simulate the interactions between two ventricles, the in-serial indirect interaction through circulatory loop and the in-parallel direct ventricular coupling.

- Utilizing the whole body model and the model for the PAL device, different designs of the PAL device are tested computationally. Based on the examination of the model parameters, practical recommendations are made on the length of the inlet graft, the resistance of the PAL device, and the compliance of the inflow chamber. 


\section{Appendix A \\ Heart Muscle Work and Energy Efficiency Index}

Heart MW is obtained by integrating along the P-V loop of the muscle pump. The area labelled $W$ in Fig. A.1 gives the external work done by the ventricle per beat. The triangular area between the systolic and diastolic pressure-volume relations and bounded by isovolumic relaxation (labelled $\mathrm{P}$ ) is potential energy $([19])$. The total area of $W$ and $P$ is called PVA (Pressure Volume Area), which has been shown to be linearly related to the oxygen consumption per beat $([19])$. Based on this knowledge, we have devised a simple index to describe ventricular efficiency in converting chemical energy of oxygen consumption to external work. We call this index the Energy Efficiency Index (EEI), which is given by: $E E I=\frac{W}{P+W}$.

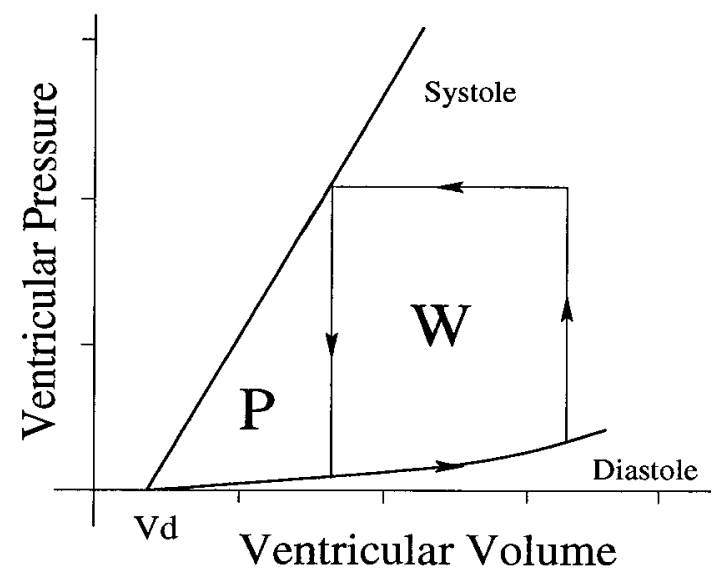

Figure A.1 Muscle Work Calculation Based on P-V Relation. 


\section{Appendix B Glossary}

\section{Abbreviations}

$H R \quad$ Heart Rate

$S V \quad$ Stroke Volume

CO Cardiac Output

$A P \quad$ Arterial Pressure

$P P \quad$ Pulse Pressure

$L V E P$ Left Ventricle Ejection Period

RVEP Right Ventricle Ejection Period

PAP Pulmonary Artery Pressure

PVP Pulmonary Artery Pressure

$M A P$ Mean Arterial Pressure

$R V E D$ End-diastolic RV volume

RVES End-systolic RV volume

LVED End-diastolic LV volume

LVES End-systolic LV volume

$R A \quad$ Right Atrium 
$R V \quad$ Right Ventricle

$L A \quad$ Left Atrium

$L V \quad$ Left Ventricle

$S V C \quad$ Superior Vena Cava

IVC Inferior Vena Cava

$P A L \quad$ Para-corporeal Artificial Lung

PAPP Pulmonary Arterial Pulse Pressure

SRVP Systolic Right Ventricular Pressure

$P Q A L$ Pulse Flow through Artificial Lung

Pressures

$P_{m c} \quad$ Myocardial pressure

$P_{v} \quad$ Intra-ventricle pressure

$P_{t h} \quad$ Intrathoracic pressure

$P_{a b} \quad$ Intra-abdominal pressure

$P_{f l} \quad$ Tissue pressure in the forelimbs

$P_{h l} \quad$ Tissue pressure in the hind-limbs

$P_{c s f} \quad$ Cerebral spinal fluid pressure

$P_{h} \quad$ Hydrostatic pressure 
$P_{a r}^{a} \quad$ Arteriolar pressure in fully-activated state

$P_{a r}^{p} \quad$ Arteriolar pressure in passive state

$P_{e x} \quad$ External pressure applied to the inflow chamber

$P_{P A} \quad$ Pulmonary arterial pressure

$P_{R A} \quad$ Right atrial pressure

$P_{R V} \quad$ Right ventricular pressure

$P_{A L} \quad$ Pressure in artificial lung chamber

\section{Volumes}

$V_{a r} \quad$ Arteriolar volume

$V_{a r, 0} \quad$ Arteriolar unstressed volume

Flows

$Q_{P A} \quad$ Pulmonary artery outflow

$Q_{A L} \quad$ Flow through the artificial lung chamber

Resistances

$R_{p} \quad$ Pulmonary valve resistance

$R_{a} \quad$ Aortic valve resistance 


$\begin{array}{ll}R_{t} & \text { tricuspid valve resistance } \\ R_{m} & \text { Mitral valve resistance } \\ R_{e p} & \text { Epicardial resistance } \\ R_{a} & \text { Large artery resistance } \\ R_{s a} & \text { Small artery resistance } \\ R_{a r} & \text { Arterioles resistance } \\ R_{c a} & \text { Capillary resistance } \\ R_{s v} & \text { Small vein resistance } \\ R_{v} & \text { Large vein resistance } \\ R_{v v} & \text { Venous valve resistance } \\ R T 1 & \text { Resistance of the inlet graft } \\ R T 2 & \text { Resistance of the outlet graft } \\ R I C & \text { Axial resistance of the inflow chamber } \\ R T I C & \text { Transmural resistance of the inflow chamber } \\ R A L & \text { Axial resistance of the artificial lung chamber }\end{array}$

\section{Compliances}

$C_{e p} \quad$ Epicardial compliance

$C_{a} \quad$ Large artery compliance 
$C_{s a} \quad$ Small artery compliance

$C_{a r} \quad$ Arterioles compliance

$C_{c a} \quad$ Capillary compliance

$C_{s v} \quad$ Small vein compliance

$C_{v} \quad$ Large vein compliance

CT1 Compliance of the inlet graft

$C T 2$ Compliance of the outlet graft

CIC Compliance of the inflow chamber

$C A L \quad$ Compliance of the artificial lung chamber

Inertances

$L_{a} \quad$ Inertance of arterial blood column

Constants

$F_{h r V} \quad$ Normalized vagal frequency controlling HR (dimensionless)

$F_{h r S} \quad$ Normalized sympathetic frequency controlling HR (dimensionless)

$\tau_{p} \quad$ Passive exponential constant $\left(m l^{-1}\right.$, Equ. 2.3) 
$F_{V A S O}$ Normalized sympathetic efferent discharge

frequency controlling vasomotor tone

(dimensionless)

$F_{C O N}$ Normalized sympathetic efferent discharge

frequency controlling cardiac contractility

(dimensionless)

$K_{a} \quad$ Scaling constant for activated arterioles

(dimensionless, Equ. 2.2)

$D_{0} \quad$ Arteriolar volume parameter ( $m l$, Equ. 2.3)

$K_{p 1} \quad$ Scaling constant for passive arterioles

(mmHg, Equ. 2.3)

$K_{p 2} \quad$ Scaling constant for passive arterioles

( $m m H g / m l^{2}$, Equ. 2.3)

Pressures are in $m m H g$, volumes in $m l$, flows in $m l / s e c$, resistances in $m m H g * s e c / m l$, compliances in $\mathrm{ml} / \mathrm{mmHg}$, and inertances in $m m H g * s e c^{2} / m l$. 


\section{References}

1. D. C. Chung, S. C. Niranjan, J. W. Clark, Jr., A. Bidani, W. E. Johnston, J. B. Zwischenberger, and D. L. Traber, A dynamic model of ventricular interaction and pericardial influence. Am. J. Physiol. 272: H2942-H2962, 1997

2. J. B. Olansen, J. W. Clark, Jr., D. Khoury, F. Ghorbel, and A. Bidani, A closed-loop model of the canine cardiovascular system that includes ventricular interaction, Computers and Biomedical Research 33, 260 - 295 (2000)

3. K. Lu, J. W. Clark, Jr., F. H. Ghorbel, D. L. Ware, and A. Bidani, A human cardiopulmonary system model applied to the analysis of the Valsalva maneuver. Am. J. Physiol. Heart Circ. Physiol. 281: H2661-H2679, 2001

4. J. Dai, J. K-J. Li, Prediction of impediment effect of cardiac contraction on coronary blood flow. FASEB J. 13: A424, 1999

5. D. A. Linkens, Identification of respiratory and cardiovascular system. In M. Naecz, editor, Control Aspects of Biomedical Engineering, page 251-265. Pergamon Press, New York, 1st edition, 1987

6. T. G. Coleman, Cardiovascular modeling, In D. G. Cramp and E. Carson, editors, The Circulatory System, chapter 6, Croom Helm, London, 1986

7. J. Beneken, Some computer models in cardiovascular research. In D. H. Bergel, editor, Cardiovascular Research, volume 2, chapter 6, pages 173-223, Academic press, New York, 1972

8. R. Skalak. Synthesis of a complete circulation, in D. H. Bergel, editor, Cardiovascular Fluid Dynamics, volume 2, chapter 19, Academic Press, New York, 1972

9. M. P. Spencer and A. B. Denison, Pulsatile blood flow in the vascular system, in P. Dow, editor, Handbook of Physiology, volume 11, chapter 25, pages 839-864, Waverly Press, Inc., Baltimore, MD, 1963

10. J. N. Amoore and W. P. Santamore, Model studies of the contribution of ventricular interdependence to the transient changes in ventricular function with respiratory efforts. Cardiovascular Research, 23(8): 683-94, 1989

11. W. P. Santamore and D. Burkhoff, Hemodynamic consequencies of ventricular interaction as assessed by model analysis, Am. J. Physiol., 260: H146-57, Jan. 1991 
12. W. P. Santamore, J. L. Heckman, and A. A. Bove, Right and left ventricular pressure-volume response to respiratory maneuvers, J. Appl. Physiol., 57: 15207,1984

13. H. H. Hardy, R. E. Collins, and R. E. Calvert, A digital computer model of the human circulatory model, Med. Biol. Eng. Comput., 20(5): 550-64, 1982

14. R. Beyar and Y. Goldstein, Model studies of the effects of the thoracic pressure on the circulation, Ann. Biomed. Eng., 15(3-4):373-83, 1987

15. J. Beneken and C. C. Rideout, The use of multiple models in cardiovascular system studies: Transport and perturbation methods, IEEE Trans. Biomed. Eng., BME-15(4): 281-289, Oct. 1968

16. K. H. Wesseling and J. J. Settels, Circulatory model of baro- and cardiopulmonary reflexes. In: Blood Pressure and Heart Rate Variability. Amersterdam, The Netherlands: IOS, 1993, p. 56-67

17. K. Sunagawa, T. Kawada, and T. Nakahara, Dynamic nonlinear vagosypathetic interaction regulating heart rate. Heart Vessels, 13:157-174, 1998.

18. R. W. Gore and M. J. Davis, Mechanics of smooth muscle in isolated single microvessels. Ann. Biomed. Eng., 12:511-520, 1984.

19. N. Westerhof, Cardiac work and efficiency, Cardiovascular Research 48 (2000) 4-7.

20. M. F. O'Rourke, Vascular impedance in studies of arterial and cardiac function, Physiol. Res. v62 NO.2 April 1982

21. M. F. O'Rourke, Mechanical Principles in Arterial Disease, Hypertension, $1995 ; 26: 2-9$

22. G. M. London, S. J. Marchais, M. E. Safar, Arterial compliance in hypertension, J Hum Hypertens 1989 Jun;3 Suppl 1:53-6

23. G. M. London, Large arteries haemodynamics: conduit versus cushioning function, Blood Press Suppl 1997;2:48-51

24. L. R. Johnson, Essential Medical Physiology, 2nd edition, Lippincott-Raven, ISBN 0-397-58401-6

25. E. Kresch, Design of a non-linear electrical model for veins( $\mathrm{PhD}$ thesis). Philadelphia, PA: University of Pennsylvania, 1968 
26. M. F. Snyder and V. V. Rideout, Computer simulation studies of the venous circulation. IEEE Trans Biomed Eng 16: 325-334, 1969

27. W. R. Lynch, J. P. Montoya, D. O. Brant, R. J. Schreiner, M. D. Iannettoni, and R. H. Barlett, Hemodynamic effect of a low-resistance artificial lung in series with the native lungs of sheep, Ann. Thorac. Surg 2000;69:351-6

28. F. Boschetti, C. E. Perlman, K. E. Cook, and L. F. Mockros, Hemodynamic effects of attachment modes and device design of a thoracic artificial lung, ASAIO J. $2000 ; 46: 42-48$

29. K. Sugi, J. Newald, L. D. Traber, J. P. Maguire, D. N. Herndon, G. Schlag, and D. L. Traber, Cardiac dysfunction after acute endotoxin administration in conscious sheep, Am. J. Physiol. 260 (Heart Circ. Physiol. 29): H1474-H1481, 1991

30. K. Slama, M Gesch, J C. Böck, S. M. Pietschmann, W Schaffartzik, and U. Pison, Unilateral lung edema: effects on pulmonary gas exchange, hemodynamics, and pulmonary perfusion distribution. J Appl Physiol 89: 1513-1521, 2000

31. S. Kono, K. Nishimura T. Nishina, T. Akamatsu, and M Komeda, Hemodynamics on abrupt stoppage of centrifugal pumps during left ventricular assist, ASAIO Journal 2000; 46:600-603

32. D. Zheng, R. N. Upton, G. L. Ludbrook, and A. Martinez, Acute cardiovascular effects of magnesium and their relationship to systemic and myocardial magnesium concentrations after short infusion in awake sheep, $\mathrm{J}$ of Pharmacology and Experimental Therapeutics, 297:1176-1183, 2001

33. M. Takagaki, D. Rottenberg, P. M. McCarthy, N. G. Smedira, R. Dessoffy, M. A. Ahmadi, D. Shoshani, and K, Fukamachi, A novel miniature ventricular assist device for hemodynamic support, ASAIO Journal 2001; 47:412-416

34. S. B. Lewis, M. L. Wong, P. E. Bannan, F.R.A.C.S., I. R. Piper, P. L. Reilly, Transcrannial doppler identification of changing autoregulatory thresholds after autoregulatory impairment, Neurosurgery, 48:369-376, 2001

35. P. B. Rich, C. A. Reickert, S.Sawada, S. S. Awad, W. R. Lynch, K. J. Johnson, R. B. Hirschl, Effects of rate and inspiratory flow on ventilator-induced lung injury, J Trauma. 2000; 49:903-911

36. P. Segers, P. Steendijk, N. Stergiopulos, N. Westerhof, Predicting systolic and diastolic aortic blood pressure and stroke volume in the intact sheep, J Biomechanics $34(2001) 41-50$ 
37. J. H. Patterson, K. F. Adams Jr., Pathophysiology of heart failure: changing perceptions, Pharmacotherapy 1996 Mar-Apr;16(2 Pt 2):27S-36S 